\title{
Reversal cycle in square Rayleigh-Bénard cells in turbulent regime
}

\author{
Andres Castillo Castellanos ${ }^{1,2}$, Anne Sergent ${ }^{2,3} \dagger$ and Maurice Rossi ${ }^{1,4}$ \\ ${ }^{1}$ Sorbonne Universités, UPMC Univ Paris 06, UMR 7190, Institut Jean Le Rond d'Alembert, \\ F-75005, Paris, France \\ ${ }^{2}$ LIMSI, CNRS, Université Paris-Saclay, F-91405 Orsay, France \\ ${ }^{3}$ Sorbonne Universités, UPMC Univ Paris 06, UFR d'Ingénierie, F-75005, Paris, France \\ ${ }^{4}$ CNRS, UMR 7190, Institut Jean Le Rond d'Alembert, F-75005, Paris, France
}

We consider long-term data from direct numerical simulations of turbulent RayleighBénard convection inside 2D square cells. For the range of Rayleigh numbers $\mathrm{Ra}=$ $10^{7}-10^{8}$ and Prandtl numbers $\operatorname{Pr}=3.0-4.3$, two types of flow regimes are observed: a regime consisting of consecutive reversals, when the global rotation switches signs; and a regime consisting of an extended cessation, when global rotation is absent. A filtering method discriminates these two regimes and allows to identify two characteristic time-scales for the former regime. A time re-scaling is then used to tune our records to a common duration thus putting into evidence a generic reversal cycle. This cycle is composed of three successive phases: acceleration, accumulation, and release including a rebound event. We complement this view in terms of global angular impulse, available mechanical energy, global kinetic energy, and their corresponding transfer rates. For a particular realisation of a reversal, each phase is described in terms of the flow patterns (large diagonal roll, counter-rotating corner-flows and thermal plumes) and tied to the corresponding energy processes. We conclude by performing linear as well as nonlinear stability studies to account for the triggering mechanism of the release.

\section{Introduction}

Decaying or stochastically forced two-dimensional turbulence in the presence of rigid boundaries is characterised by self-organised coherent structures. For a square domain (Clercx et al. 1998; Molenaar et al. 2004), a spontaneous spin-up is observed which leads to the formation of a single vortex structure. This structure can persist for very long periods of time, before suddenly breaking and re-organising itself, in some cases with a reversed rotation sense. A similar process occurs in the geomagnetic field under the form of polarity switches (Wicht et al. 2009; Valet et al. 2012). In turbulent Rayleigh-Bénard (RB) convection experiments, this phenomenon is also observed: a large scale circulation (LSC), commonly referred to as the wind, changes sign intermittently. Several models have been proposed to describe this process in RB convection through either stochastic differential equations (Sreenivasan et al. 2002; Benzi 2005; Brown \& Ahlers 2007; Podvin \& Sergent 2015) or phenomenological and physically motivated assumptions (Araujo et al. 2005; Resagk et al. 2006; Brown \& Ahlers 2007).

The LSC structure, its global properties and the transition between dominant flow structures are found to be dependent on the cavity shape (Grossmann \& Lohse 2003; Xi \& Xia 2008b; van der Poel et al. 2011). Inside cylindrical cells, a change of sign of the

$\dagger$ Email address for correspondence: anne.sergent@limsi.fr 
LSC occurs either by a rotation-led reversal through an azimuthal rotation of the nearvertical circulation plane known as azimuthal meandering, or by a cessation-led reversal through the breakdown of the existing LSC before re-organising in a different spatial direction (see, for instance Niemela et al. (2001); Sreenivasan et al. (2002); Brown \& Ahlers (2007); Funfschilling et al. (2008); Xi \& Xia (2008a,b)). One approach to separate rotation-led from cessation-led reversal events consists in restricting the experimental study to a square box of small aspect ratio in the transversal direction (Xia et al. 2003; Sugiyama et al. 2010; Wagner \& Shishkina 2013; Ni et al. 2015). Another viewpoint uses two-dimensional direct numerical simulations (Sugiyama et al. 2010; Petschel et al. 2011; Chandra \& Verma 2011; Verma et al. 2015; Podvin \& Sergent 2015) since rotation-led reversals are not possible in such a configuration. However, it is not entirely clear whether $2 \mathrm{D}$ reversals and cessation-led reversals correspond to the same phenomenon. Sugiyama et al. (2010) have identified a region in the (Ra, Pr) space in which reversal events are observed experimentally inside quasi-two-dimensional cells as well as numerically in two-dimensional simulations. For this range of ( $\mathrm{Ra}, \mathrm{Pr})$, the flow inside a square cell is mainly composed of a large diagonal roll and two counter-rotating corner-rolls. (Chandra \& Verma 2013; Sugiyama et al. 2010) pointed out the feeding of corner-rolls by plumes detached from horizontal boundary layers. Both papers proposed that the growth of corner-rolls ended by a sudden LSC transition.

The presence of such coherent structures has been investigated by computing the first Fourier modes (Chandra \& Verma 2011; Verma et al. 2015), or by obtaining these modes from a proper orthogonal decomposition (Bailon-Cuba et al. 2010; Podvin \& Sergent 2015). Coherent structures are actually associated with a sum of various such modes: a large-scale monopole, a quadrupole and a vertically or horizontally stacked dipole. A study of the transition sequences between these first Fourier modes indicated the presence of a reversal path (Petschel et al. 2011). To analyse such a process, one could adopt the perspective used in geomagnetic fields combining a careful selection of reversal records as well as a time rescaling (Valet et al. 2012; Lhuillier et al. 2013). In geomagnetism, this method led to the definition of three successive phases: a precursory event, a polarity switch and a rebound.

Another viewpoint is based on energetical considerations. For instance available potential energy is key to understand how mechanical energy is transported, stored, and dissipated in RB convection (Winters et al. 1995; Hughes et al. 2013). This approach could make more precise the idea of an avalanche mechanism (mentioned in Sreenivasan et al. (2002)), due to a localised accumulation of energy, which increases local gradients until a certain threshold is reached and energy is expelled as a single burst.

In the present paper, we propose for $\mathrm{RB}$ convection, a formulation similar to the one proposed in geomagnetism (Valet et al. (2012)): the main objective is to establish the existence of a generic reversal cycle and to identify in this cycle three phases (release, accumulation, and acceleration). This analysis combines a statistical analysis with a physical approach relying on the angular momentum as well as kinetic and potential energy to highlight the underlying physical mechanisms. In addition, we identify flow patterns corresponding to each phase of the generic cycle by using a conditional averaging. A threshold state in generic reversal cycles is identified from which the release is inevitable.

The paper is organised as follows. Section 2 introduces the model equations and global quantities: global angular impulse, available mechanical energy and corresponding conversion rates. A brief description of the numerical method and the spatial resolution is presented in section 3. In section 4, a filtering method is proposed that identifies two regimes, and then allows to perform a statistical study of reversals. The dynamics of a 
generic reversal mechanism is described as composed by three phases in section 5 . These results are then analysed in terms of coherent flow structures, physical mechanisms in section 6 for particular realisations. In section 7, a stability analysis is applied on the generic cycle. Section 8 contains a brief comparison of the present analysis with previous works. Finally, some prospective works are mentioned in conclusion.

\section{Model equations and analysis tools}

Consider a fluid contained in a square cell, cooled at the top with constant temperature $\mathrm{T}_{\text {top }}$ and heated at the bottom with constant temperature $\mathrm{T}_{\text {bot }}>\mathrm{T}_{\text {top }}$. The flow equations are based on the Boussinesq approximation. The flow regime is defined as a function of the Rayleigh and Prandtl numbers,

$$
\operatorname{Ra} \equiv \frac{g H^{3} \beta\left(\mathrm{T}_{\text {bot }}-\mathrm{T}_{\text {top }}\right)}{\kappa \nu}, \quad \operatorname{Pr} \equiv \frac{\nu}{\kappa}
$$

where $g$ denotes gravity, $H$ the cell height and $\beta, \kappa, \nu$ are respectively volumetric thermal expansion, thermal diffusivity and kinematic viscosity coefficients. The values of (Ra, Pr) used for direct numerical simulations (DNS) correspond to a weakly turbulent flow regime where reversals have been reported (Sugiyama et al. 2010). As far as notations are concerned, $x$ (resp. $u$ ) and $y$ (resp. $v$ ) stand for the horizontal and vertical directions (resp. velocities). Coordinate vector $\vec{x}=(x, y)$ is equal to $(0,0)$ at the cavity centre. One introduces the reduced temperature $\theta(\vec{x}, t) \equiv\left(\mathrm{T}-\mathrm{T}_{0}\right) /\left(\mathrm{T}_{\text {bot }}-\mathrm{T}_{\text {top }}\right)$, with $\mathrm{T}_{0} \equiv\left(\mathrm{T}_{\text {bot }}+\mathrm{T}_{\text {top }}\right) / 2$ as well as the only vorticity component $\omega(\vec{x}, t) \equiv \partial_{x} v-\partial_{y} u$. For a field $a(\vec{x}, t)$, the fields $\bar{a}(\vec{x})$ and $\sigma(a)(\vec{x})$ denote the time average and standard deviation computed using the full long-term time series. In addition, quantity $\langle a\rangle_{\text {vol }}(t)$ stands for the volume average of $a(\vec{x}, t)$.

Based on the cell height $H$ as characteristic length scale and $\frac{\kappa}{H} \mathrm{Ra}^{0.5}$ as velocity scale, the dimensionless velocity $\vec{u}=(u, v)$ and reduced temperature $\theta$ satisfy the dimensionless system of equations

$$
\left\{\begin{aligned}
\nabla \cdot \vec{u} & =0, \\
\partial_{t} \vec{u}+\nabla \cdot[\vec{u} \otimes \vec{u}] & =-\nabla p+\operatorname{PrRa}^{-0.5} \nabla^{2} \vec{u}+\operatorname{Pr} \theta \vec{e}_{y}, \\
\partial_{t} \theta+\nabla \cdot[\vec{u} \theta] & =\operatorname{Ra}^{-0.5} \nabla^{2} \theta
\end{aligned}\right.
$$

No-slip condition for the velocity field is ensured on walls. On top (resp. bottom) walls, one imposes $\theta=-0.5$ (resp. $\theta=0.5$ ) while adiabaticity $\partial_{x} \theta=0$ is satisfied on side-walls. From now on, quantities are written in dimensionless form only.

\subsection{Global angular impulse}

The global angular momentum

$$
\mathrm{L}_{2 \mathrm{D}}(t) \equiv-\frac{1}{2} \int \vec{x}^{2} \omega(\vec{x}, t) \mathrm{d} x \mathrm{~d} y
$$

serves as a measure of organised rotation (see for instance Molenaar et al. (2004)). Figure 1 shows a time series of the normalised angular momentum $\mathrm{L}_{2 \mathrm{D}} / \overline{\left|\mathrm{L}_{2 \mathrm{D}}\right|}$. Two different regimes are observed. Blue areas correspond to periods of time where $\mathrm{L}_{2} \mathrm{D}$ changes sign spontaneously over time: positive (resp. negative) peaks in $\mathrm{L}_{2 \mathrm{D}}$ alternate that are associated to a dominant counter-clockwise (resp. clockwise) central vortex. The blue areas consisting of a sequence of consecutive transitions is hereafter called the consecutive reversal (CR) regime. Outside this regime, the LSC is no longer well-defined 


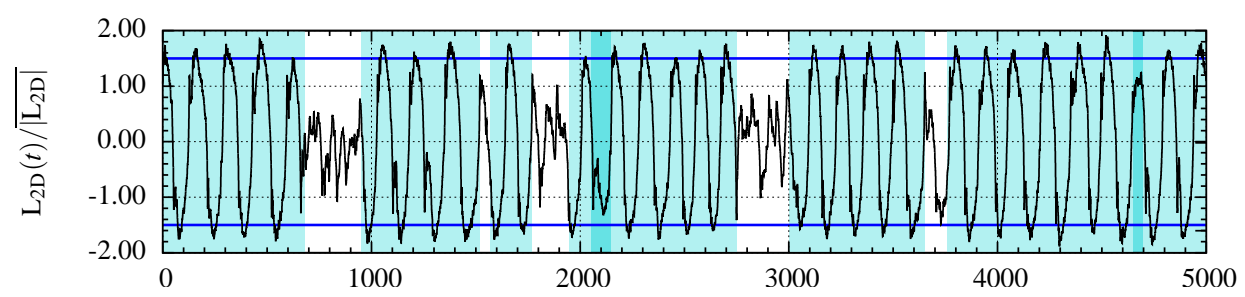

Figure 1. Time evolution of $\mathrm{L}_{2 \mathrm{D}}(t) / \overline{\left|\mathrm{L}_{2 \mathrm{D}}\right|}$ shown for $\left(\mathrm{Ra}=5 \cdot 10^{7}, \operatorname{Pr}=3\right)$. Light blue areas correspond to a consecutive reversal (CR) regime, while blank areas correspond to an extended cessation (EC) regime. Some events (darker blue areas) may not be clearly assigned to CR régime, see text. The two continuous lines correspond to the thresholds used by the filtering procedure. Value of the normalized standard deviation: $\sigma\left(\left|\mathrm{L}_{2 \mathrm{D}}\right|\right) / \overline{\left|\mathrm{L}_{2 \mathrm{D}}\right|}=0.499$.

and one observes an extended cessation. Such complementary region is denoted here as the extended cessation (EC) regime.

In order to differentiate in a precise manner the $\mathrm{CR}$ regime from the $\mathrm{EC}$ regime, a filtering algorithm has been devised which is modeled after (Lhuillier et al. 2013; Podvin \& Sergent 2015). We identify the set of consecutive times $r_{i}$ at which $\mathrm{L}_{2 \mathrm{D}}$ changes sign. The time interval $\left[r_{i}, r_{i+1}\right]$ is considered to be inside the CR regime if, during this interval, the value of $\left|\mathrm{L}_{2 \mathrm{D}}\right|$ reaches at least once the threshold value $\overline{\left|\mathrm{L}_{2 \mathrm{D}}\right|}+\sigma\left(\left|\mathrm{L}_{2 \mathrm{D}}\right|\right.$ ) (light blue area in figure 1). A time interval where such threshold is not reached can be of two kinds corresponding to the darker blue areas or the white areas in figure 1 . The first kind is sandwiched between two CR intervals and corresponds to a "rogue" reversal, which likely belongs to the $\mathrm{CR}$ regime but has been filtered out by our criterion (the criteria for the selection of events in the CR regime is rather stringent as seen from the "rogue" events displayed in figure 1). The second type, displayed in white, corresponds to an extended cessation.

For any interval $\left[r_{i}, r_{i+1}\right]$, its duration $\tau_{1, i} \equiv r_{i+1}-r_{i}$ is also computed. When both intervals $\left[r_{i-1}, r_{i}\right]$ and $\left[r_{i}, r_{i+1}\right]$ are inside a CR regime, the duration $\tau_{d, i}$ of the jump occurring around time $r_{i}$ between a clockwise and counter-clockwise central vortex or vice versa can be evaluated. It is computed by identifying the times located just before and just after time $r_{i}$ such that $\left|\mathrm{L}_{2 \mathrm{D}}\right|$ reaches the threshold value $\overline{\left|\mathrm{L}_{2 \mathrm{D}}\right|}-\sigma\left(\left|\mathrm{L}_{2 \mathrm{D}}\right|\right) \cdot \tau_{d, i}$ is simply the time lapse between these two events.

The change of the global angular momentum $\mathrm{L}_{2 \mathrm{D}}$ may be better understood considering the relation directly obtained from the governing equations (2.2),

$$
\frac{\mathrm{dL}_{2 \mathrm{D}}}{\mathrm{dt}}=\mathrm{M}+\mathrm{I}_{\mathrm{a}}-\mathrm{I}_{\mathrm{b}} \quad\left\{\begin{array}{l}
\mathrm{M}(t) \equiv \frac{1}{2} \operatorname{Pr} \int \vec{x}^{2} \partial_{x} \theta \mathrm{d} x \mathrm{~d} y \\
\mathrm{I}_{\mathrm{a}}(t) \equiv \operatorname{PrRa}^{-0.5} \oint[\vec{x} \cdot \vec{n}] \omega \mathrm{d} l \\
\mathrm{I}_{\mathrm{b}}(t) \equiv \frac{1}{2} \operatorname{PrRa}^{-0.5} \oint \vec{x}^{2} \vec{n} \cdot \nabla \omega \mathrm{d} l
\end{array}\right.
$$

where $\vec{n}$ stands for the outwards unit normal vector to the domain boundary and $\mathrm{d} l$ for a contour line differential element. Note that it is assumed that line integrals are performed in a counterclockwise direction. The angular momentum thus evolves because of a bulk forcing term $\mathrm{M}(t)$ known as the input torque (Molenaar et al. 2004) and two boundary integrals terms $\mathrm{I}_{\mathrm{a}}(t)$ and $\mathrm{I}_{\mathrm{b}}(t)$. $\mathrm{I}_{\mathrm{b}}(t)$ is close but not identical to the integrated vorticity flux over the domain boundary. For a square cavity, the boundary term $\mathrm{I}_{\mathrm{a}}(t)$ simplifies to $\mathrm{I}_{\mathrm{a}}=\frac{1}{2} \mathrm{PrRa}^{-0.5} \oint \omega \mathrm{d} l$. Vorticity on the boundary is related to the friction exerted by the fluid on the walls. This integral $\mathrm{I}_{\mathrm{a}}(t)$ is thus quantifying friction along the boundary. 

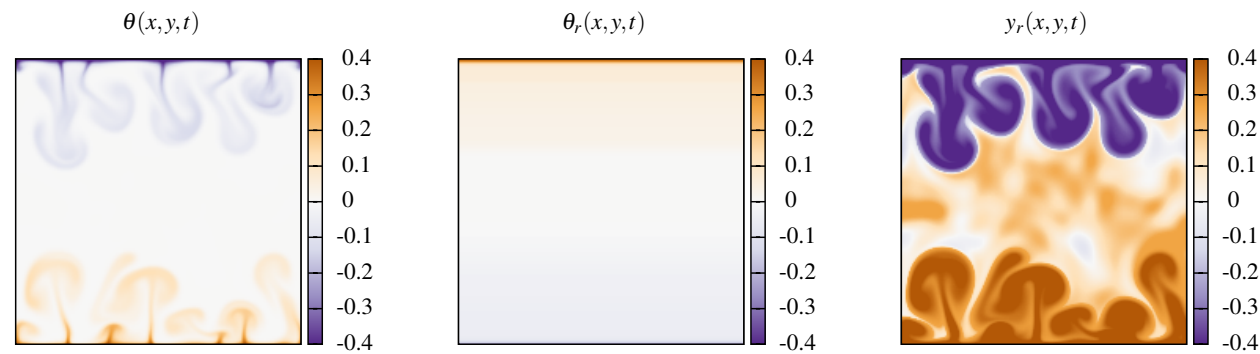

Figure 2. Temperature field $\theta(x, y, t)$ at a given instant $t$ (left), the corresponding background state $\theta_{r}(y, t)$ (center) and height $y_{r}(x, y, t)$ (right) for a square RB cell.

\subsection{Mechanical energy balance}

Other global quantities are useful to characterise at each time the instantaneous state of the system: the global kinetic energy $\mathrm{E}_{\text {kin }}(t) \equiv \frac{1}{2} \int \vec{u}^{2} \mathrm{~d} x \mathrm{~d} y$ and the global potential energy $\mathrm{E}_{\text {pot }}(t) \equiv-\operatorname{Pr} \int y \theta \mathrm{d} x \mathrm{~d} y$. This latter quantifies the energy amount required to bring all fluid particles against gravity from their position at time $t$ to the reference level $y=0$. However, one can introduce a more pertinent instantaneous quantity namely the available potential energy (Sutherland 2010), which is defined here below. For a given time $t$, the fluid is characterized by an instantaneous temperature field $\theta(x, y, t)$ with a lower (resp. upper) bound at $\theta_{\min }\left(\right.$ resp. $\left.\theta_{\max }\right)$. Let us consider a one-to-one mapping $\left(x_{r}(x, y), y_{r}(x, y)\right)$ from the square onto the square. This may be interpreted as a reordering of fluid particles inside the square cell. Rearranging modifies the temperature field leading to a new field $\theta_{r}(x, y, t)$ but this process is an adiabatic one i.e. $\theta_{r}\left(x_{r}, y_{r}, t\right)=\theta(x, y, t)$. As a consequence, the probability distribution function $(\mathrm{PDF})$ of temperature in the rearranged state is identical to the PDF in the instantaneous state. The potential energy of the rearranged state can be measured. Among the set of such mappings, there exists a subset which corresponds to the lowest potential energy. It is easy to understand that all mappings belonging to this subset have identical rearranged temperature field $\theta_{r}(y, t)$ with no $x$ dependency and increasing (i.e. density decreasing) monotonically with height (figure 2). This field characterizes the background state. Although $y_{r}(x, y, t)$ is not a simple function of $(x, y)$, the above remark implies that $y_{r}$ is a one-to one function of $\theta$ for a mapping in this subset. In practice (see Tseng \& Ferziger (2001)), the field $\theta_{r}(y, t)$ is computed by the following procedure. First, the PDF of the instantaneous temperature at time $t$ which is denoted by $P(\theta)$, is directly evaluated numerically within the interval $\left[\theta_{\min }, \theta_{\max }\right]$ since $\theta(x, y, t)$ is known on the whole square box. Second, the conservation of temperature PDF with rearrangement imposes that $y_{r}(\theta)$ be evaluated as a cumulative density function

$$
y_{r}(\theta)-y_{b o t}=y_{r}(\theta)-y_{r}\left(\theta_{\text {min }}\right)=\int_{\theta_{\min }}^{\theta} P(\theta) \mathrm{d} \theta .
$$

This relation depends a priori on the domain geometry. For a square box of unit size, however, the proportionality is factor is reduced to unity. Third, function $\theta_{r}(y, t)$ is then obtained by a simple inversion of $y_{r}(\theta, t)$.

The background state is characterized by the lowest potential energy that can be reached by an adiabatic process starting from the instantaneous temperature field $\theta(x, y, t)$. This quantity called the background potential energy is equal to $\mathrm{E}_{\text {bpot }} \equiv-\operatorname{Pr} \int y_{r}(x, y, t) \theta(x, y, t) \mathrm{d} x \mathrm{~d} y$. By a simple change of variable and using 
adiabaticity $\theta_{r}\left(x_{r}, y_{r}, t\right)=\theta(x, y, t)$, one gets

$$
\mathrm{E}_{\mathrm{bpot}}=-\operatorname{Pr} \int y_{r} \theta_{r}\left(y_{r}\right) \mathrm{d} x_{r} \mathrm{~d} y_{r}=-\operatorname{Pr} \int y_{r} \theta_{r}\left(y_{r}\right) \mathrm{d} y_{r} .
$$

The difference $\mathrm{E}_{\text {apot }}(t) \equiv \mathrm{E}_{\text {pot }}(t)-\mathrm{E}_{\text {bpot }}(t)>0$ in potential energy between the instantaneous state and its background companion is called the available potential energy and represents the potential energy which could be effectively transformed from the instantaneous field $\theta(x, y, t)$ into motion (Lorenz 1955; Winters et al. 1995).

In analogy with $\mathrm{L}_{2 \mathrm{D}}$, the process may be better grasped by considering the evolution of the energies $\mathrm{E}_{\mathrm{kin}}, \mathrm{E}_{\mathrm{pot}}, \mathrm{E}_{\mathrm{apot}}$, through some exact relations (see Winters et al. (1995); Hughes et al. (2013)). For the kinetic energy $\mathrm{E}_{\mathrm{kin}}$, the following relation holds $\left(e_{i j}\right.$ denotes the symmetric velocity gradient tensor)

$$
\frac{\mathrm{dE}_{\mathrm{kin}}}{\mathrm{dt}}=\operatorname{PrRa}^{-0.5}\left[\Phi_{y}-\epsilon\right], \quad\left\{\begin{array}{c}
\Phi_{y} \equiv \mathrm{Ra}^{0.5}\langle v \theta\rangle_{\mathrm{vol}} \\
\epsilon \equiv\langle\nabla \vec{u}: \nabla \vec{u}\rangle_{\mathrm{vol}}=2\left\langle e_{i j} e_{i j}\right\rangle_{\mathrm{vol}}
\end{array}\right.
$$

The first bulk term $\Phi_{y}(t)$ is a convective heat flux. More precisely let us introduce the volume-averaged Nusselt number $\mathrm{Nu}_{\mathrm{vol}} \equiv \mathrm{Ra}^{0.5}\langle v \theta\rangle_{\mathrm{vol}}-\left\langle\partial_{y} \theta\right\rangle_{\mathrm{vol}}$. It is easily found that, for RB cells, $\Phi_{y}(t)=\mathrm{Nu}_{\mathrm{vol}}(t)-1$. The second bulk term $\epsilon(t)>0$ stands for the viscous dissipation rate. Finally one may write

$$
\frac{\mathrm{dE}_{\mathrm{kin}}}{\mathrm{dt}}=\operatorname{PrRa}^{-0.5}\left[\mathrm{Nu}_{\mathrm{vol}}-(\epsilon+1)\right]
$$

The potential energy $\mathrm{E}_{\text {pot }}$ verifies instead the relation

$$
\frac{\mathrm{dE}_{\mathrm{pot}}}{\mathrm{dt}}=\operatorname{PrRa}^{-0.5}\left[-\mathrm{Nu}_{\mathrm{vol}}+\Phi_{b 1}\right]
$$

which contains the bulk term $\mathrm{Nu}_{\mathrm{vol}}$ and a boundary term

$$
\Phi_{b 1}(t) \equiv-\oint y[\vec{n} \cdot \nabla \theta] \mathrm{d} l
$$

quantifying the conversion rate to $\mathrm{E}_{\text {pot }}$ from external sources. More precisely, let us introduce the Nusselt number $\mathrm{Nu}_{\text {top }}(t) \equiv-\int \partial_{y} \theta \mathrm{d} x$ evaluated at the top $y=0.5$ as well as the Nusselt number $\mathrm{Nu}_{\text {bot }}(t) \equiv-\int \partial_{y} \theta \mathrm{d} x$ evaluated at the bottom plates $y=$ -0.5 . For the present square cell, one easily verifies that $\Phi_{b 1}=\frac{1}{2}\left(\mathrm{Nu}_{\text {top }}+\mathrm{Nu}_{\text {bot }}\right)$ and consequently

$$
\frac{\mathrm{dE}_{\mathrm{pot}}}{\mathrm{dt}}=\operatorname{PrRa}^{-0.5}\left[-\mathrm{Nu}_{\mathrm{vol}}+\frac{1}{2}\left(\mathrm{Nu}_{\text {top }}+\mathrm{Nu}_{\text {bot }}\right)\right]
$$

Finally, the evolution equation for the available potential energy $\mathrm{E}_{\mathrm{bpot}}$ reads as

$$
\frac{\mathrm{dE}_{\mathrm{bpot}}}{\mathrm{dt}}=\operatorname{PrRa}^{-0.5}\left[\Phi_{d}-\Phi_{b 2}\right], \quad\left\{\begin{array}{l}
\Phi_{d} \equiv\left\langle\frac{\partial y_{r}}{\partial \theta} \boldsymbol{\nabla} \theta \cdot \nabla \theta\right\rangle_{\mathrm{vol}}=\left\langle\nabla y_{r} \cdot \nabla \theta\right\rangle_{\mathrm{vol}} \\
\Phi_{b 2} \equiv \oint y_{r}[\vec{n} \cdot \nabla \theta] \mathrm{d} l
\end{array}\right.
$$

where the bulk term $\Phi_{d}(t)$ quantifies the energy conversion rate due to diapycnal mixing. Since by definition $\frac{\partial y_{r}}{\partial \theta}>0, \Phi_{d}(t)$ is bound to be positive. The boundary term $\Phi_{b 2}$ provides the conversion rate from external sources. For the present RB cells, since $y_{r}(x, 1 / 2, t)=-1 / 2$ and $y_{r}(x,-1 / 2, t)=1 / 2$ and because adiabaticity of side walls it is clear that $\Phi_{b 2}(t)=\Phi_{b 1}(t)$. Finally by substracting equation 2.8 by equation 2.11 , one gets

$$
\frac{\mathrm{dE}_{\mathrm{apot}}}{\mathrm{dt}}=\operatorname{PrRa}^{-0.5}\left[\mathrm{Nu}_{\mathrm{bot}}+\mathrm{Nu}_{\mathrm{top}}-\mathrm{Nu}_{\mathrm{vol}}-\Phi_{d}\right]
$$




\begin{tabular}{ll|llll}
$\mathrm{Pr}$ & $\mathrm{Ra}$ & Time & Events & $\overline{\mathrm{Nu}}$ & \%Diff \\
3.0 & $10^{7}$ & 9,600 & 48 & 12.5 & 0.30 \\
& $3 \cdot 10^{7}$ & 9,600 & 77 & 17.6 & 0.53 \\
& $5 \cdot 10^{7}$ & 9,600 & 83 & 20.7 & 0.69 \\
& $10^{8}$ & 12,000 & 83 & 25.6 & 1.44 \\
\hline \multirow{2}{*}{4.3} & $3 \cdot 10^{7}$ & 19,000 & 22 & 18.4 & 0.24 \\
& $5 \cdot 10^{7}$ & 29,000 & 161 & 21.0 & 0.70 \\
& $10^{8}$ & 19,000 & 156 & 25.9 & 1.38
\end{tabular}

TABle 1. For various Prandtl and Rayleigh numbers, the table provides the simulation length in convective time units, the number of reversal events, the average Nusselt number and maximum relative difference between $\overline{\mathrm{Nu}}_{\mathrm{vol}}, \overline{\mathrm{Nu}}_{\mathrm{top}}, \overline{\mathrm{Nu}}_{\mathrm{bot}}, \overline{\mathrm{Nu}}_{\theta}$, and $\overline{\mathrm{Nu}}_{\epsilon}$.

\section{Numerical method}

Simulations are carried out using a finite volume code using a semi-implicit scheme based on Bell-Colella-Glaz advection scheme (Bell et al. 1989), and a pressure-correction scheme for the velocity-pressure coupling, with a global second order precision. Numerical implementation is done using BASILISK $\mathrm{C}$, details of which can be found in Popinet (2016). Simulations listed in table 1 have been performed on a uniform Cartesian grid with 512 points in each direction, with a variable time-step that verifies the condition $\mathrm{CFL}<0.5$. In the most unfavorable case $\left(\mathrm{Ra}=10^{8}, \mathrm{Pr}=4.3\right)$ the thermal boundary layers contain 10 points along the vertical direction.

Spatial resolution is verified evaluating numerical convergence of time-averaged Nusselt numbers obtained by different methods (Stevens et al. 2010). Note that to perform the averaging, statistical sampling is obtained at regular intervals. We compare $\overline{\mathrm{Nu}}_{\mathrm{vol}}, \overline{\mathrm{Nu}}_{\text {top }}$ and $\overline{\mathrm{Nu}}_{\text {bot }}$ to the Nusselt numbers obtained from the thermal and viscous dissipations

$$
\overline{\mathrm{Nu}}_{\theta} \equiv\langle\overline{\nabla \theta \cdot \nabla \theta}\rangle_{\mathrm{vol}}, \quad \overline{\mathrm{Nu}}_{\epsilon} \equiv \bar{\epsilon}+1
$$

All these quantities should be equal (Shraiman \& Siggia 1990). The value of $\overline{\mathrm{Nu}}$ shown in table 1 is the average value of $\overline{\mathrm{Nu}}_{\mathrm{vol}}, \overline{\mathrm{Nu}}_{\text {top }}, \overline{\mathrm{Nu}}_{\text {bot }}, \overline{\mathrm{Nu}}_{\theta}, \overline{\mathrm{Nu}}_{\epsilon}$ while the maximum relative difference between any of them is shown as \%Diff. These values converge within $2 \%$ of $\overline{\mathrm{Nu}}$ for all $(\mathrm{Ra}, \mathrm{Pr})$ presented. We have also verified that our numerical results are wellconverged in a completely different way. This check has been performed by comparing results of the probability density functions (PDF) of the time interval $\tau_{1}$ obtained by our code against benchmark results. This computation requires to get long term simulations and was done for $\left(\mathrm{Ra}=5 \cdot 10^{7}, \mathrm{Pr}=4.3\right)$ since such parameter values had already been computed by a spectral code (Podvin \& Sergent 2015). The data for this check are postponed at the end of section 4 .

\section{Temporal analysis and statistical characterisation}

In the present work, we focus on turbulent RB systems for which flow reversals are observed. For $\operatorname{Pr}=3.0$, this dynamics is associated with the interval $\operatorname{Ra} \in\left[5 \cdot 10^{6}, 3 \cdot 10^{8}\right]$. For $\operatorname{Pr}=4.3$, it corresponds to the interval $\mathrm{Ra} \in\left[3 \cdot 10^{7}, 4 \cdot 10^{8}\right]$ (Sugiyama et al. 2010). Note that, these boundaries are not clearly established. For instance, some transitions were found for very long runs at $\left(\operatorname{Pr}=4.3, \mathrm{Ra}=10^{7}\right)$ (not presented here) but it is difficult to assert whether or not the few cycles observed correspond to an established statistical steady state or to a transient behaviour. In the following, we consider only 


\begin{tabular}{ll|lllll|lll}
$\operatorname{Pr}$ & $\mathrm{Ra}$ & $p_{c r}$ & $p_{r r}$ & $p_{e c}$ & $\mathrm{Pr}$ & $\mathrm{Ra}$ & $p_{c r}$ & $p_{r r}$ & $p_{e c}$ \\
3.0 & $10^{7}$ & $85 \%$ & $1 \%$ & $14 \%$ & 4.3 & - & - & - & - \\
& $3 \cdot 10^{7}$ & $73 \%$ & $3 \%$ & $24 \%$ & & $3 \cdot 10^{7}$ & $95 \%$ & $1 \%$ & $4 \%$ \\
& $5 \cdot 10^{7}$ & $77 \%$ & $3 \%$ & $20 \%$ & & $5 \cdot 10^{7}$ & $83 \%$ & $1 \%$ & $16 \%$ \\
& $10^{8}$ & $96 \%$ & $4 \%$ & $0 \%$ & & $10^{8}$ & $89 \%$ & $2 \%$ & $9 \%$
\end{tabular}

TABle 2. Probabilities as a function of Ra and Pr. $p_{c r}$ (resp. $p_{e c}$ ) denotes the probability that the system be inside the CR (resp. EC) regime. $p_{r r}$ denotes the probability of a "rogue" reversal..

values inside the aforementioned range for which the number of events is large enough (see table 1): simulations are performed from 9,600 to 29,000 convective time units (see table 1), which gives from 50 to 160 events (except for $\operatorname{Pr}=4.3$ and $\mathrm{Ra}=3 \cdot 10^{7}$ which is situated close to the boundary region where the reversal dynamics is established).

For a given couple ( $\mathrm{Ra}, \mathrm{Pr}$ ), one computes the percentage of time or equivalently the probability that the system be in one of the three states: $p_{c r}$ in the $\mathrm{CR}$ regime, $p_{e c}$ in the EC regime and $p_{r r}$ inside a "rogue" reversal. The probability $p_{r r}$ of rogue events is always of a few percents (see table 2). For both $\operatorname{Pr}=3.0$ and $\operatorname{Pr}=4.3$, in the interval where the CR regime is observed, $p_{c r}$ first decreases and then increases with increasing Ra (see table 2).

The PDFs of $\tau_{1}$ and $\tau_{d}$ are measured based on the full simulation length and shown on figure 3 (resp. figure 4) for $\operatorname{Pr}=4.3$ (resp. $\operatorname{Pr}=3$ ). Using the filtering method of section 2.1, we separated the PDF of $\tau_{1}$ into three contributions: one for intervals inside the $\mathrm{CR}$ regime (color blue), one for intervals from the EC regime (color red) and one corresponding to rogue events (color purple). This PDF shows that the distribution of $\tau_{1}$ is not peaked inside the $\mathrm{CR}$ regime: intervals may have different durations. This is also valid for the EC regime. For $\operatorname{Pr}=4.3$, a similar probability distribution of $\tau_{1}$ is observed for both values of Ra (figure 3 ) and a characteristic time-scale $\tau_{c} \approx 60$ exists which separates EC from CR regimes. For $\operatorname{Pr}=3.0$, a change in the PDFs of $\tau_{1}$ and $\tau_{d}$ is observed as we increase the values of Ra (figure 4). For the lowest Ra displayed, a characteristic time $\tau_{c}$ cannot be clearly defined. For the highest $\mathrm{Ra}$ displayed $\mathrm{Ra}=10^{8}$, the EC regime completely disappears. For intermediate Ra, reversal events become evenly distributed over a narrow band of $\tau_{1}$ (see figure 4 center and right) and a clear separation of time-scales between the EC and CR regimes is observed at $\tau_{c} \approx 50$ which is at least one order of magnitude larger than the large eddy turn-over time, $\tau_{E} \equiv 4 \pi / \overline{\left|\omega_{c}\right|}\left(\omega_{c}\right.$ denoting vorticity measured at the cavity center). The PDF of the inter-switch intervals observed for cylindrical convection cell experiments is an exponential distribution (Sreenivasan et al. (2002)). It is not seen here (figures 3 and 4) illustrating the fact that rotation-led reversals are not present here contrary to cylindrical cells.

From the PDF of transition durations $\tau_{d}$ for CR regime only (see figures 3 and 4 bottom), the peak value tends to increase as $\mathrm{Ra}$ is increased for both $\operatorname{Pr}=3.0$ and $\operatorname{Pr}=4.3$. Concerning the numerical check, the average value $\left.\bar{\tau}_{1}\right|_{\text {cr }}$ during the CR regime and the average duration $\overline{\tau_{d}}$ of transition were both found to be in good agreement with published results for $\left(\mathrm{Ra}=5 \cdot 10^{7}, \operatorname{Pr}=4.3\right):\left.\overline{\tau_{1}}\right|_{\mathrm{cr}}=146$ and $\overline{\tau_{d}}=11.5$ convective time units (Podvin \& Sergent 2015).

\section{Dynamics of the generic reversal}

We have shown above that reversals cycles have different durations $\tau_{1, i}$. A simple time-rescaling, however, can be used to identify features common to all reversal cyles. 

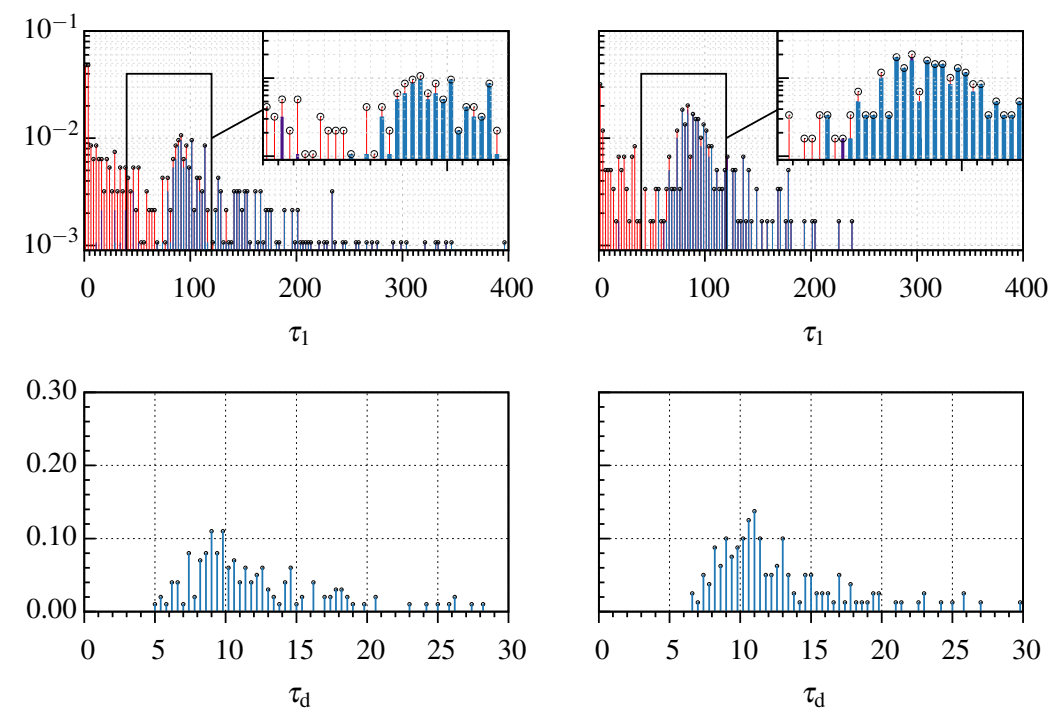

Figure 3. Probability density function (PDF) of $\tau_{1}$ (top) and $\tau_{\mathrm{d}}$ (bottom) for $\mathrm{Pr}=4.3$, (left figure) $\mathrm{Ra}=5 \cdot 10^{7}$, (right figure) $\mathrm{Ra}=10^{8}$. The $\mathrm{PDF}$ value is represented by $\circ$ marks. For $\tau_{1}$ it is the sum of three conditional PDFs: one in (- $(-)$ corresponding to the CR regime, a smaller one in (— corresponding to the EC regime.
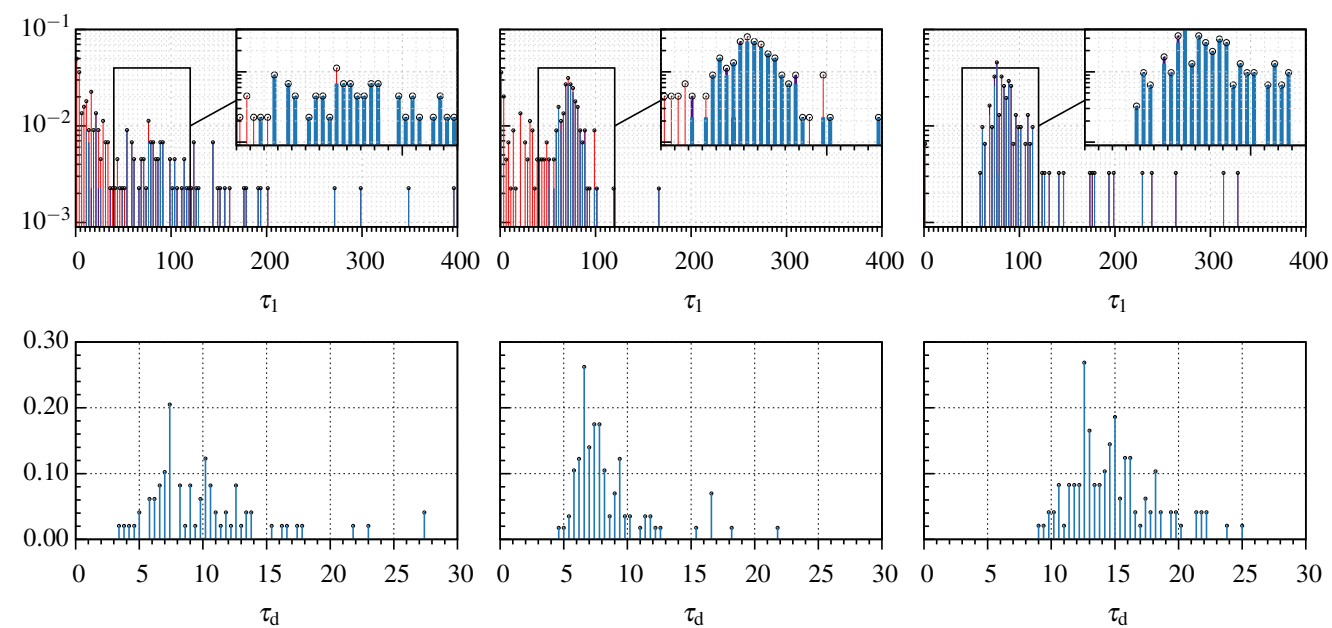

Figure 4. Probability density function (PDF) for $\tau_{1}$ (top) and $\tau_{\mathrm{d}}$ (bottom) for $\operatorname{Pr}=3.0$ and $\mathrm{Ra}=10^{7}$ (left), $\mathrm{Ra}=5 \cdot 10^{7}$ (centre) and $\mathrm{Ra}=10^{8}$ (right). Layout is similar to figure 4.

\subsection{Averaging procedure and generic reversal as function of $(\mathrm{Ra}, \mathrm{Pr})$}

In order to identify similarities between different intervals in the CR regime, the following procedure is proposed to treat time-series of global quantities such as $\mathrm{L}_{2 \mathrm{D}}$, $\mathrm{E}_{\mathrm{kin}}$, and $\mathrm{E}_{\text {apot }}$. Once the intervals $\left[r_{i}, r_{i+1}\right]$ inside the $\mathrm{CR}$ regime are properly identified, all these intervals with $\mathrm{L}_{2 \mathrm{D}}>0$ (resp. $\mathrm{L}_{2 \mathrm{D}}<0$ ) are stacked together so that they have a common origin at $r_{i}$ (resp. $r_{i+1}$ ). If the time axis of each interval is re-scaled by $\overline{\tau_{1}}$, one obtains figure 5 left. If the time axis of each interval is rescaled by its particular duration $\tau_{1, i}$, these curves display a consistent dynamical pattern (see figure 5 centre). 

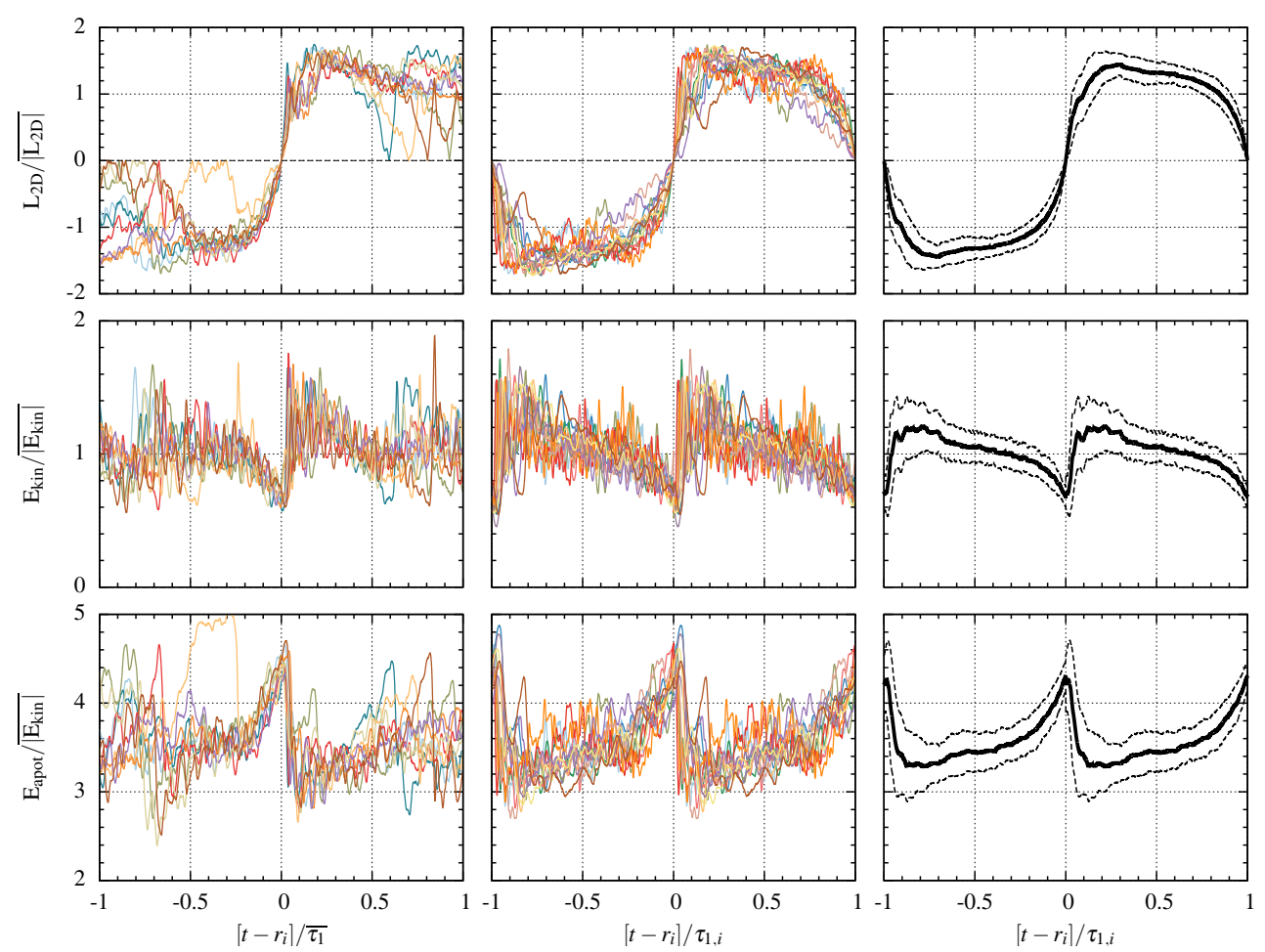

Figure 5. From top to bottom: Normalised global angular impulse $\mathrm{L}_{2 \mathrm{D}} / \overline{\left|\mathrm{L}_{2 \mathrm{D}}\right|}$, normalised kinetic energy $E_{\text {kin }} / \overline{\left|E_{\text {kin }}\right|}$, and normalised available potential energy $E_{\text {apot }} / \overline{\left|E_{\text {kin }}\right|}$ for $\left(\mathrm{Ra}=5 \cdot 10^{7}, \mathrm{Pr}=4.3\right)$. Left panel: Each reversal cycle is centred and its time is rescaled by $\bar{\tau}_{1}$ (only 10 reversals are displayed and each colour is a different reversal); Centre panel: Each reversal cycle is centred and its time is rescaled by $\tau_{1, i}$; Right panel: Average value of rescaled curves obtained from the complete time-series (thick lines) and curves corresponding to one standard deviation (dashed lines).

Note that, while figure 5 displays only 10 reversals to avoid cluttered graphs, these events displayed are considered as representative of the entire set. Obviously, all of the events inside the CR regime are taken into account in our procedure but "rogue reversal" events are not. This procedure is similar to one used in the study of statistical properties of magnetic switches in the geodynamo problem (Valet et al. 2012; Lhuillier et al. 2013). For a sufficiently large number of recorded events, the average over these rescaled curves is expected to remove the noisy dynamics and to represent a generic reversal cycle (figure 5 right). This averaging technique, once applied to $\mathrm{E}_{\mathrm{kin}}$ and $\mathrm{E}_{\text {apot }}$, recovers the evolution of mechanical energies during the generic reversal cycle.

Figure 6 shows the $\mathrm{L}_{2 \mathrm{D}} / \overline{\left|\mathrm{L}_{2 \mathrm{D}}\right|}$ curves for generic reversal cycle at $\operatorname{Pr}=3.0$ : as we increase $\mathrm{Ra}$, the reversal cycle becomes more regular and the band representing the standard deviation narrows. The same averaging procedure can be applied to instantaneous temperature and velocity fields in order to obtain the evolution of a conditionally averaged temperature $\theta^{o}(x, y, t)$ and velocity $\vec{u}^{o}(x, y, t)$ fields during the reversal cycle (e.g. figure 7 for $\left.\left(\mathrm{Ra}=5 \cdot 10^{7}, \mathrm{Pr}=3.0\right)\right)$. Despite fluctuations between various realisations, dominant and persistent structures appear at specific times of the generic cycle. 

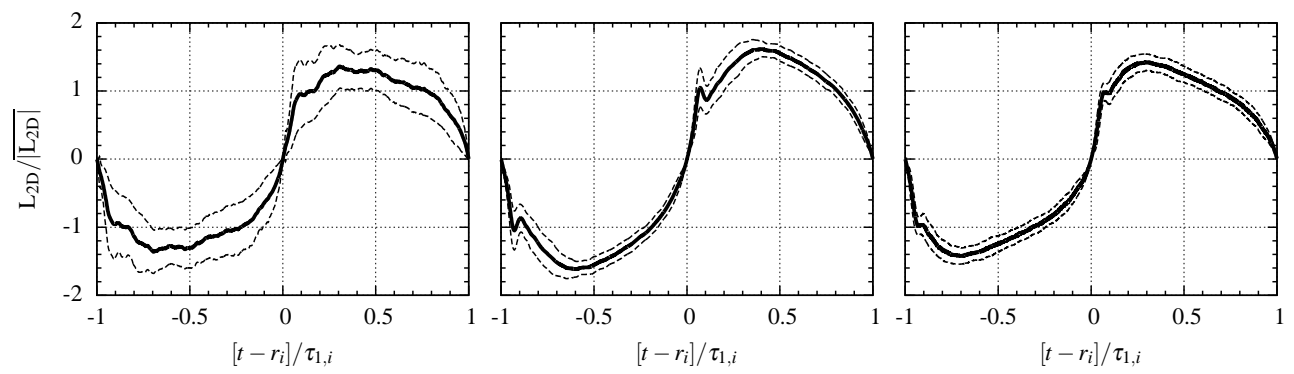

Figure 6. Curves corresponding to reversals for $\operatorname{Pr}=3.0$ as obtained using the procedure described in figure 5 . The average $\mathrm{L}_{2 \mathrm{D}} / \overline{\left|\mathrm{L}_{2 \mathrm{D}}\right|}$ is shown in thick lines and one standard deviation in dashed lines. From left to right: a) $\left.\mathrm{Ra}=10^{7}, \mathrm{~b}\right) \mathrm{Ra}=5 \cdot 10^{7}$, and c) $\mathrm{Ra}=10^{8}$.

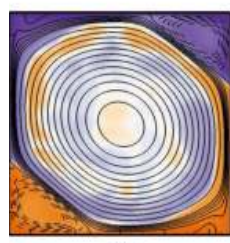

(i)

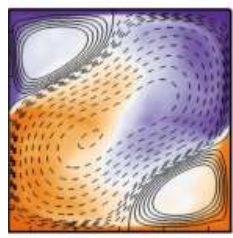

(vi)

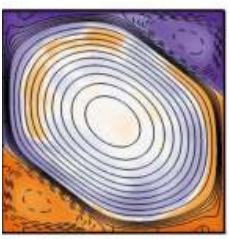

(ii)

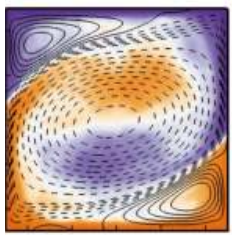

(vii)

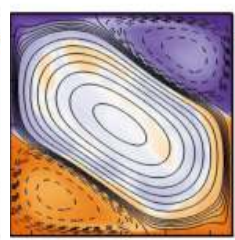

(iii)

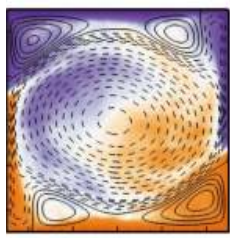

(viii)

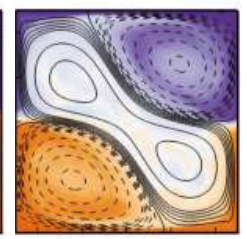

(iv)

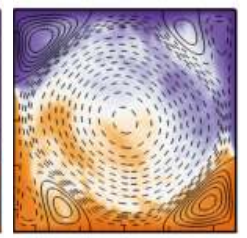

(ix)

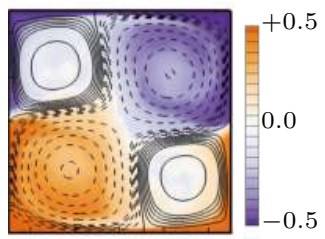

(v)

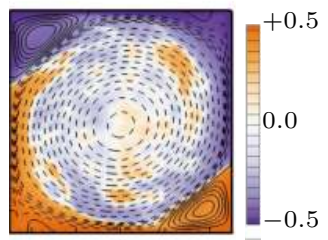

$(\mathrm{x})$

FiguRE 7 . Conditionally averaged fields $\vec{u}^{o}(x, y, t)$ and $y_{r}\left(\theta^{o}(x, y, t)\right)$ at different instants during the generic reversal cycle for $\left(\mathrm{Ra}=5 \cdot 10^{7}, \mathrm{Pr}=3.0\right)$. Fields are obtained as the ensemble average over 83 particular reversal cycles. Streamlines of the velocity field $\vec{u}^{\circ}$ are superposed over the color map of field $y_{r}\left(\theta^{\circ}\right)$. Solid and dashed streamlines indicate the two senses of rotation.

\subsection{Phases of reversal cycle}

In the phase space $\left(\mathrm{L}_{2 \mathrm{D}} / \overline{\left|\mathrm{L}_{2 \mathrm{D}}\right|}, \mathrm{E}_{\mathrm{kin}} / \overline{\left|\mathrm{E}_{\mathrm{kin}}\right|}, \mathrm{E}_{\text {apot }} / \overline{\left|\mathrm{E}_{\mathrm{kin}}\right|}\right)$, let us consider the generic reversal cycle (figure 8). Consecutive instants (a)-(b)-(c)-(d)-(e) pinpoints particular dynamical times: $\mathrm{L}_{2 \mathrm{D}}=0$ at instant (a); $\mathrm{E}_{\mathrm{kin}}$ reaches a local maximum at instant (b), $\mathrm{E}_{\text {kin }}$ reaches a local minimum at instant $(\mathrm{c}) ; \mathrm{E}_{\text {apot }}$ reaches its minimum at instant $(\mathrm{d})$; $\left|\mathrm{L}_{2 \mathrm{D}}\right|$ reaches its maximum at instant (e). Points (a')-(b')-(c')-(d')-(e') are similar but correspond to an opposite rotation sign. Based on these instants, three successive phases are identified for the generic reversal cycle. They are called accumulation, release and acceleration.

The accumulation phase is located between points (e') and (a). It is characterised by a steady accumulation of $\mathrm{E}_{\text {apot }}$ and a progressive decay of $\left|\mathrm{L}_{2 \mathrm{D}}\right|$ and $\mathrm{E}_{\mathrm{kin}}$. This phase ends when $\mathrm{E}_{\text {apot }}$ reaches a maximum and $\mathrm{E}_{\mathrm{kin}}$ a minimum (figure 8). In terms of generic velocity field $\vec{u}^{o}(x, y, t)$, a central vortex is present during this phase (see figures 7 i to iii) until the global rotation switches signs at point (a) i.e. $\mathrm{L}_{2 \mathrm{D}}=0$ (figure 7 iv).

The release phase located between points (a) and (d), is defined by a sudden exchange from $E_{a p o t}$ to $E_{k i n}$. It can be split in three sub-steps. The first step from point (a) to point (b) contains a rapid increase of $\mathrm{E}_{\text {kin }}$ to a maximum value and a rapid decrease of 

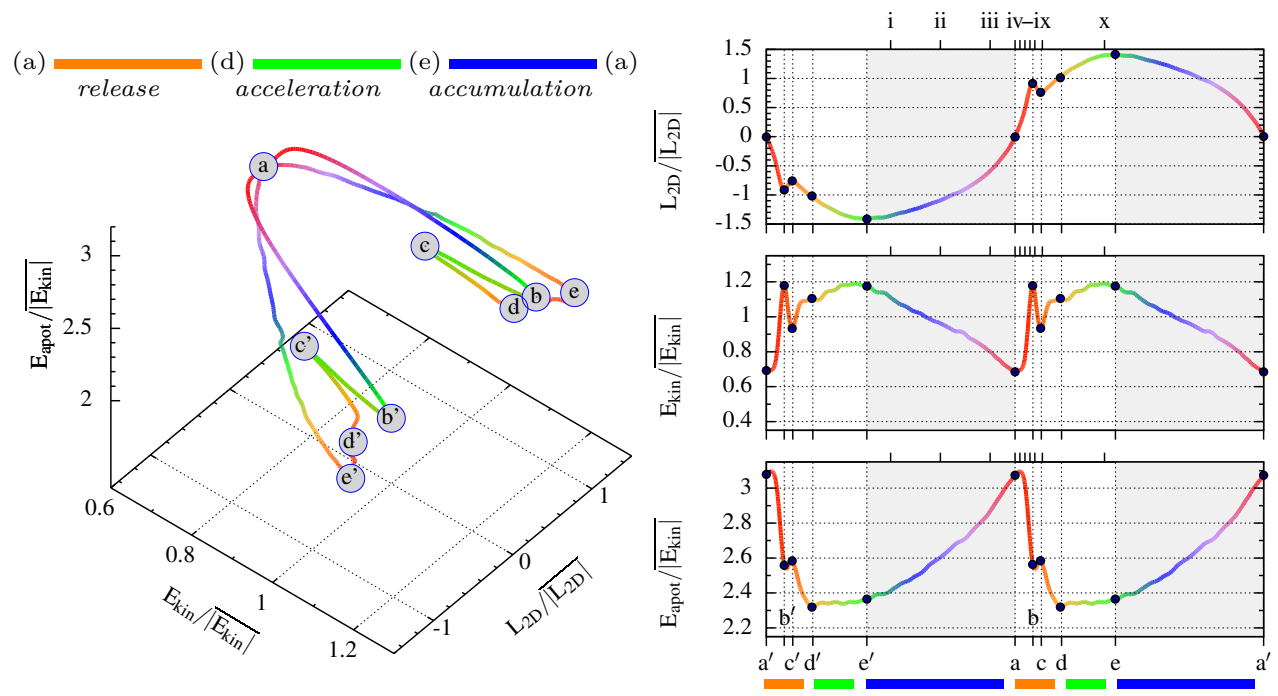

Figure 8. Generic reversal cycle for $\left(\mathrm{Ra}=5 \cdot 10^{7}, \mathrm{Pr}=3.0\right)$. Left panel: system trajectory in phase space $\left(\mathrm{L}_{2 \mathrm{D}} / \overline{\left|\mathrm{L}_{2 \mathrm{D}}\right|}, \mathrm{E}_{\text {kin }} / \overline{\left|\mathrm{E}_{\text {kin }}\right|}, \mathrm{E}_{\text {apot }} / \overline{\left|\mathrm{E}_{\text {kin }}\right|}\right)$; Right panel from top to bottom: average curves for $\mathrm{L}_{2 \mathrm{D}} / \overline{\left|\mathrm{L}_{2 \mathrm{D}}\right|}, \mathrm{E}_{\text {kin }} / \overline{\left|\mathrm{E}_{\text {kin }}\right|}$ and $\mathrm{E}_{\text {apot }} / \overline{\left|\mathrm{E}_{\text {kin }}\right|}$. Marks (i-x) and (a-e) indicate particular instants (see text for explanations). The accumulation phase is shaded and indicated by a blue colour bar. The release (resp. acceleration) phase is indicated by an orange (resp. green) colour bar. The same colour code is used in the phase space trajectory position.
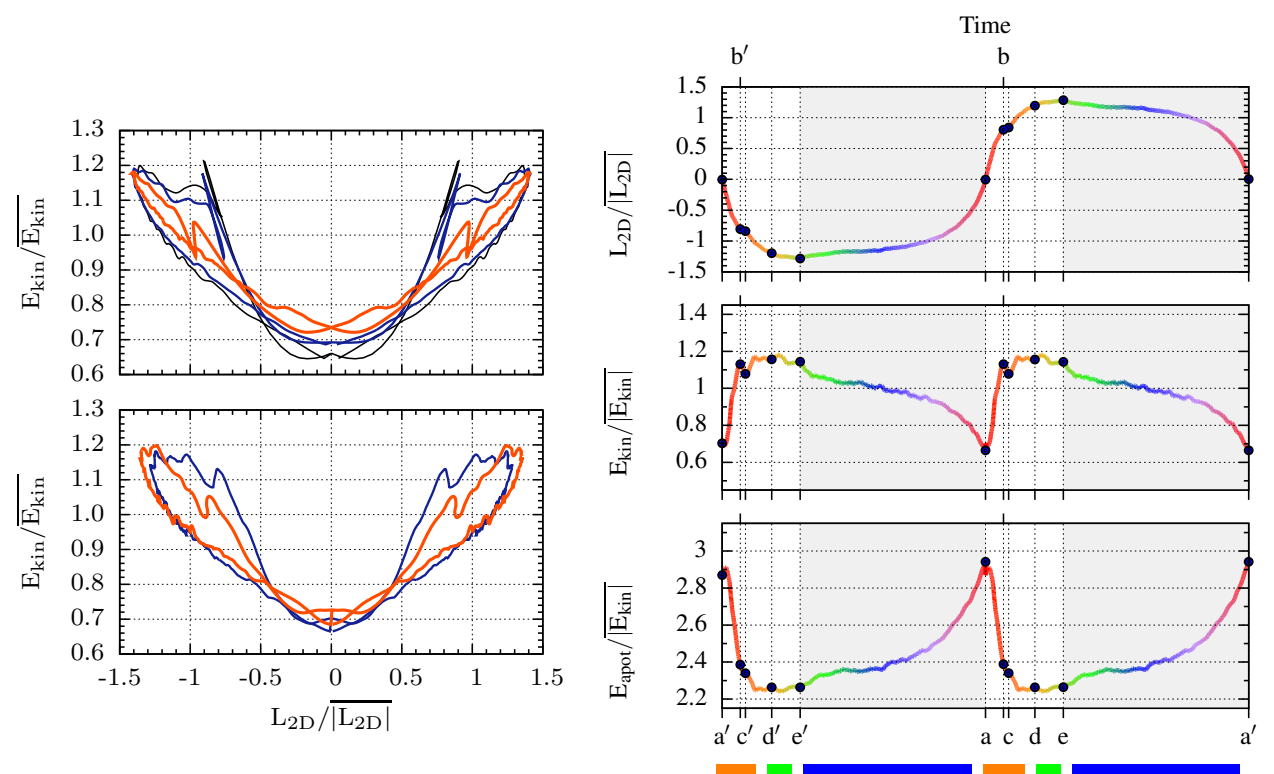

FIGURE 9. Left panel: generic reversal curves in the plane $\left(\mathrm{L}_{2 \mathrm{D}} / \overline{\left|\mathrm{L}_{2 \mathrm{D}}\right|}, \mathrm{E}_{\mathrm{kin}} / \overline{\left|\mathrm{E}_{\mathrm{kin}}\right|}\right)$. Each curve corresponds to a different $\mathrm{Ra}: \mathrm{Ra}=3 \cdot 10^{7}(\longrightarrow), \mathrm{Ra}=5 \cdot 10^{7}\left((-)\right.$, and $\mathrm{Ra}=10^{8}(\longrightarrow)$. Top figure correspond to $\operatorname{Pr}=3.0$ and bottom one to $\operatorname{Pr}=4.3$. Right panel: average curves during one cycle for $\mathrm{L}_{2 \mathrm{D}} / \overline{\left|\mathrm{L}_{2 \mathrm{D}}\right|}, \mathrm{E}_{\mathrm{kin}} / \overline{\left|\mathrm{E}_{\mathrm{kin}}\right|}$ and $\mathrm{E}_{\text {apot }} / \overline{\left|\mathrm{E}_{\mathrm{kin}}\right|}$ for $\left(\mathrm{Ra}=5 \cdot 10^{7}, \mathrm{Pr}=4.3\right)$. Figures are displayed as in figure 8 . 
$\mathrm{E}_{\text {apot}}$. It corresponds to figures $7 \mathrm{v}$ to vii. A second step follows from points (b) to (c) in which $\mathrm{E}_{\text {kin }}$ suddenly decreases and $\mathrm{E}_{\text {apot }}$ remains almost constant. This is associated with figures 7 viii to ix. After these two steps referred to as a rebound, a new increase of $E_{\text {kin }}$ is observed from points (c) to (d) concomitantly with a decrease of $E_{\text {apot }}$ until it reaches its minimum value.

Finally, the acceleration phase is located between points (d) and (e) and is characterised by an increase of $\left|\mathrm{L}_{2 \mathrm{D}}\right|$ and $\mathrm{E}_{\text {kin }}$ to peak values, whereas $\mathrm{E}_{\text {apot }}$ remains almost constant. During this period, the flow reorganises gradually into a single dominant vortex (figure $7 \mathrm{x})$.

For the $(\mathrm{Ra}, \mathrm{Pr})$ considered inside the $\mathrm{CR}$ regime, we are able to recover a generic reversal cycle expressed in terms of the available mechanical energy (figure 9). Similarities between these curves for different $(\mathrm{Ra}, \mathrm{Pr})$ suggest an equivalent underlying mechanism behind flow reversals, even if the intensity of the rebound decreases with Pr. For ( $\mathrm{Ra}=$ $\left.5 \cdot 10^{7}, \operatorname{Pr}=3.0\right)$, the accumulation phase lasts longer $(60 \%)$, while the release and acceleration phases have shorter and similar durations (respectively $18 \%$ and $22 \%$ of the reversal cycle). For the range of Ra considered these proportions are similar: for instance, the accumulation, release, and acceleration are observed to last $75 \%, 13 \%$, and $12 \%$ of the reversal cycle for $\left(\mathrm{Ra}=10^{8}, \mathrm{Pr}=3.0\right)$.

\section{Dynamics of a particular reversal}

From now on, we focus on a single value of $(\mathrm{Ra}, \mathrm{Pr})\left(\mathrm{Ra}=5 \cdot 10^{7}, \mathrm{Pr}=3.0\right)$ in order to explore the nature of the reversal dynamics. To look at the small-scales effects, the analysis below considers particular realisations of reversal cycles rather than conditionally averaged fields.

For each particular reversal cycle, we define similarly to the generic curve, consecutive instants $\left(\mathrm{a}_{p}\right)-\left(\mathrm{b}_{p}\right)-\left(\mathrm{c}_{p}\right)-\left(\mathrm{d}_{p}\right)-\left(\mathrm{e}_{p}\right)$ which pinpoints the dynamical times: $\mathrm{L}_{2 \mathrm{D}}=0$ at instant $\left(\mathrm{a}_{p}\right)$; $\mathrm{E}_{\text {kin }}$ reaches a local maximum at instant $\left(\mathrm{b}_{p}\right), \mathrm{E}_{\mathrm{kin}}$ reaches a local minimum at instant $\left(\mathrm{c}_{p}\right)$; $\mathrm{E}_{\text {apot }}$ reaches its minimum at instant $\left(\mathrm{d}_{p}\right) ;\left|\mathrm{L}_{2 \mathrm{D}}\right|$ reaches its maximum at instant $\left(\mathrm{e}_{p}\right)$.

\subsection{Time evolution of the available mechanical energy and flow structures}

The spatial distribution of mechanical energy can be linked to flow structures observed during different phases of a reversal cycle. At the beginning of the accumulation phase (point $\left(\mathrm{e}_{p}{ }^{\prime}\right)$ ), a large diagonal vortex with small counter-rotating corner-flows is observed (top figure 10). On the one hand, it corresponds to a state of maximum kinetic energy condensed inside the central vortex (bottom figure 10). On the other hand, the integrand $\operatorname{Pr} \int\left(y_{r}-y\right)$ of $\mathrm{E}_{\text {apot }}$ is mostly distributed inside the corner-flows or along thin thermal boundary layers (top figure 11). The field $\nabla y_{r} \cdot \nabla \theta$ is used to highlight the contour of thermal plumes (bottom figure 11). This field is related to the spatial distribution of $\Phi_{d}$ (see equation 2.11). Small-scale thermal plumes are observed to be detached from the thermal boundary layers. They are then swept by the central vortex. Plumes are channelled into the corner-flows, directly or after having been advected along the side-walls. The progressive growth of corner-flows is illustrated on figures 10 and 11 . This is consistent with previous observations by (Sugiyama et al. 2010) that tied the time evolution of the corner-flows height. The steady increase in $\mathrm{E}_{\text {apot }}$ coincides with a deceleration of the central vortex and to a build-up of thermal energy inside the cornerflows. Indeed, let us compute the contributions to $\mathrm{E}_{\text {apot }}$ due to the region of thermal boundary layers (BL) and the bulk. The hot and cold BL are taken to have a constant 


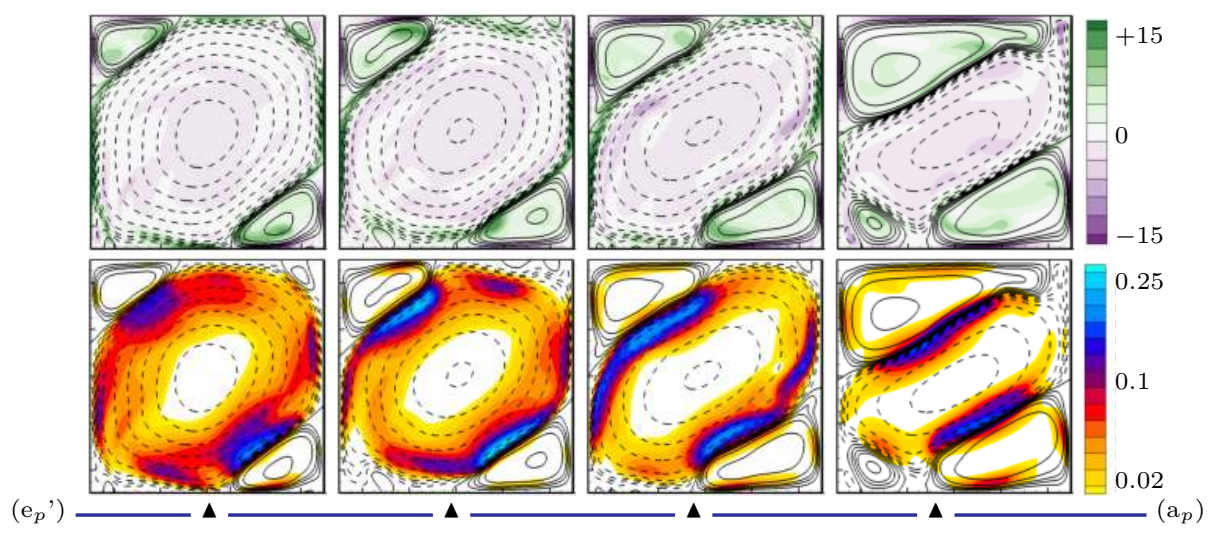

FiguRE 10. Instantaneous fields for a particular reversal cycle during the accumulation phase at regular time intervals at $\left(\mathrm{Ra}=5 \cdot 10^{7}, \mathrm{Pr}=3.0\right)$. The first snapshot follows point $\left(\mathrm{e}_{p}{ }^{\prime}\right)$ the last precedes point $\left(a_{p}\right)$. Streamlines are superposed either to vorticity $\omega$ (top figures), or to kinetic energy $\frac{1}{2} u_{i} u_{i}$ (bottom figures).
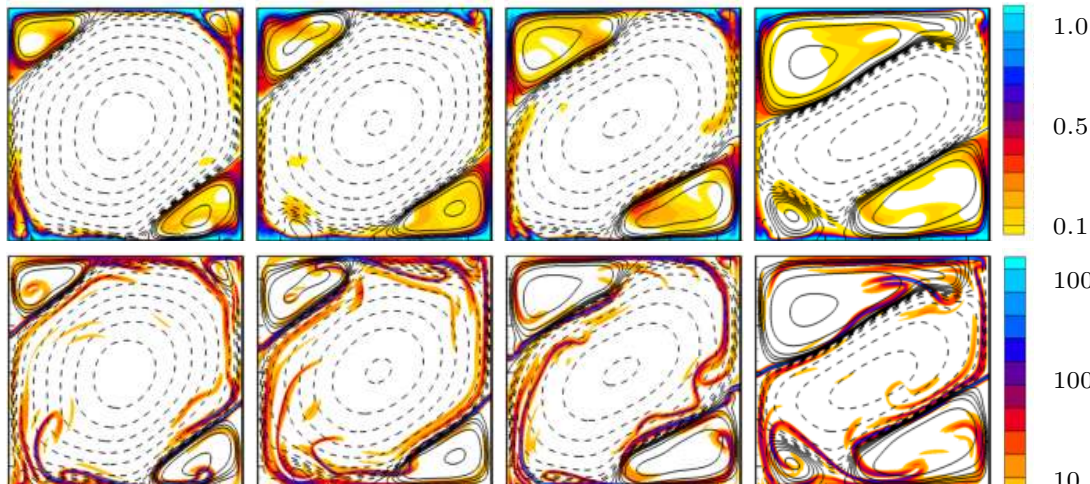

1000

$\left(\mathrm{e}_{p}{ }^{\prime}\right)$

$\Delta$

$\Delta$

FIGURE 11. Instantaneous fields for a particular reversal cycle during the accumulation phase. They are identical to those of figure 10 but streamlines are superposed either to the field $\operatorname{Pr}\left(y_{r}-y\right) \theta$ (top figures) or to the field $\nabla y_{r} \cdot \nabla \theta$ (bottom figures). Snapshots correspond to the same instants as in figure 10 .

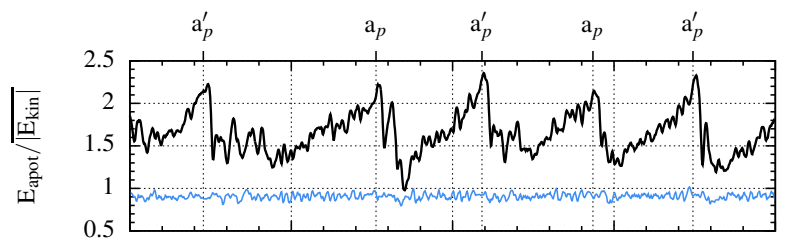

outside BL

inside BL

FiguRE 12. Evolution of $\mathrm{E}_{\text {apot }}$ contained within the thermal boundary layers $(-)$ and outside $(\longrightarrow)$ at $\left(\mathrm{Ra}=5 \cdot 10^{7}, \mathrm{Pr}=3.0\right)$.

thickness $\delta_{\theta}^{-1}=2 \overline{\mathrm{Nu}}$. Contributions inside both BL (figure 12) amount to 30-40\% of $\overline{\mathrm{E}_{\mathrm{apot}}}$ and are fairly constant in time: standard deviation is less than $1 \%$. On the contrary, contributions to $\mathrm{E}_{\mathrm{apot}}$ from outside $\mathrm{BL}$ are directly influenced by the reversal cycle: a steady increase is observed during the accumulation phase until the release phase (figure 12) . This suggests that the energy exchange observed during the release takes place only inside the bulk, while the boundary layers seem to be largely unaffected by the reversals. 

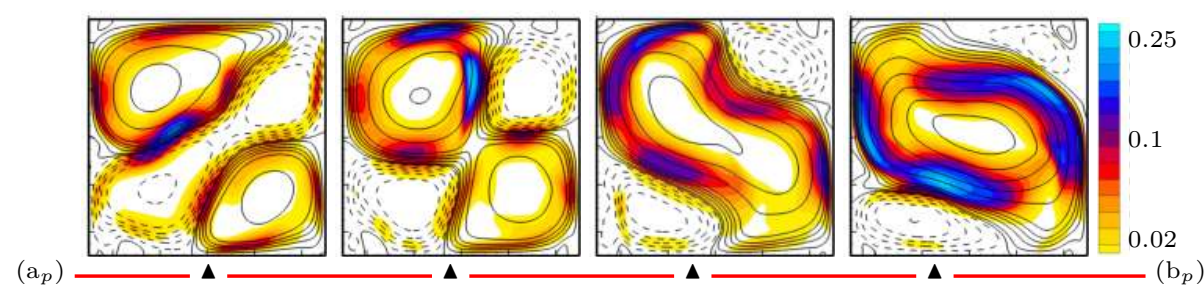

FiguRe 13. Instantaneous fields for a particular reversal cycle during the early release phase (point $\left.\left(\mathrm{a}_{p}\right)-\left(\mathrm{b}_{p}\right)\right)$ at regular time intervals $\left(\mathrm{Ra}=5 \cdot 10^{7}, \mathrm{Pr}=3.0\right)$. Streamlines are superposed to the field $\frac{1}{2} u_{i} u_{i}$. First snapshot follows point $\left(\mathrm{a}_{p}\right)$, last precedes point $\left(\mathrm{b}_{p}\right)$.
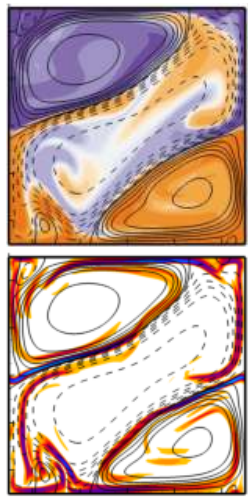

$\left(a_{p}\right)$
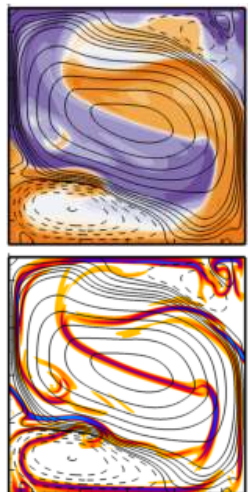

$\left(\mathrm{b}_{p}\right)$
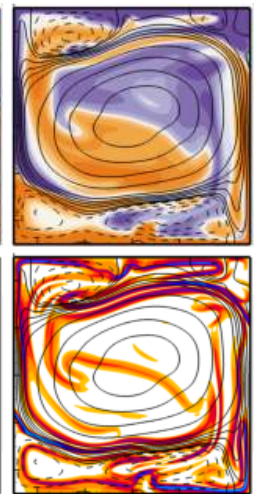

$\left(c_{p}\right)$

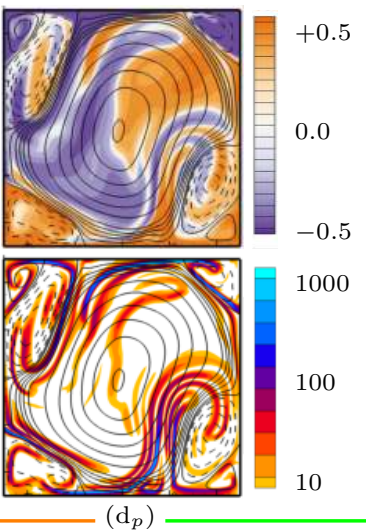

$\left(\mathrm{d}_{p}\right)$

FiguRE 14. Instantaneous fields for a particular reversal cycle during the release phase at $\left(\mathrm{Ra}=5 \cdot 10^{7}, \mathrm{Pr}=3.0\right)$. Snapshots correspond to points $\left(\mathrm{a}_{p}\right)$ to $\left(\mathrm{d}_{p}\right)$. Streamlines are superposed to either the field $y_{r}(x, z, t)$ (top figures), or to field $\nabla y_{r} \cdot \nabla \theta$ (bottom figures).

During the first part of the rebound (points $\left(a_{p}\right)$ to $\left(b_{p}\right)$ ), the opposing corner-flows have become large and strong enough to deform and finally split the central vortex (figure 13): opposing corner-flows then connect and form a single vortex (with opposite rotation with respect to the previous LSC). This allows the thermal energy stored inside corners to be rapidly released and transformed into kinetic energy. By the end of this exchange, the amount of thermal energy $E_{\text {apot }}$ stored outside the thermal boundary layers will be halved (figure 12). The complete rebound period (point $\left(\mathrm{a}_{p}\right)-\left(\mathrm{c}_{p}\right)$ ) can be better illustrated by highlighting the role of the thermal plumes and boundary layers. To do so, one uses $y_{r}(x, y, t)$ which is a bijective function of temperature, and function $\nabla y_{r} \cdot \nabla \theta$ in figure 14. Once the vortex reconnection has taken place, the blobs of hot (resp. cold) fluid which are now inside the main central vortex, are allowed to travel upwards (resp. downwards) directly into the bulk. This results in the exchange of potential energy (contained inside small scale structures) into kinetic energy (contained inside the central vortex). The newly formed circulation proceeds to rotate and is able to advect thermal blobs against the action of buoyancy forces (as seen between points $\left(b_{p}\right)$ and $\left(c_{p}\right)$ figure 14). Simultaneously as the thermals are released into the bulk, the surface separating hot and cold fluid increases which reinforces the mixing process (bottom figures 14).

The acceleration phase is illustrated on figure 15. Self-organisation of the LSC takes place, during which the kinetic energy and the angular impulse arrive to peak values. During that phase, the bulk contains less and less plumes: temperature becomes nearly homogeneous. By the end of this phase the flow settles to a large diagonal roll with small corner-flows. 


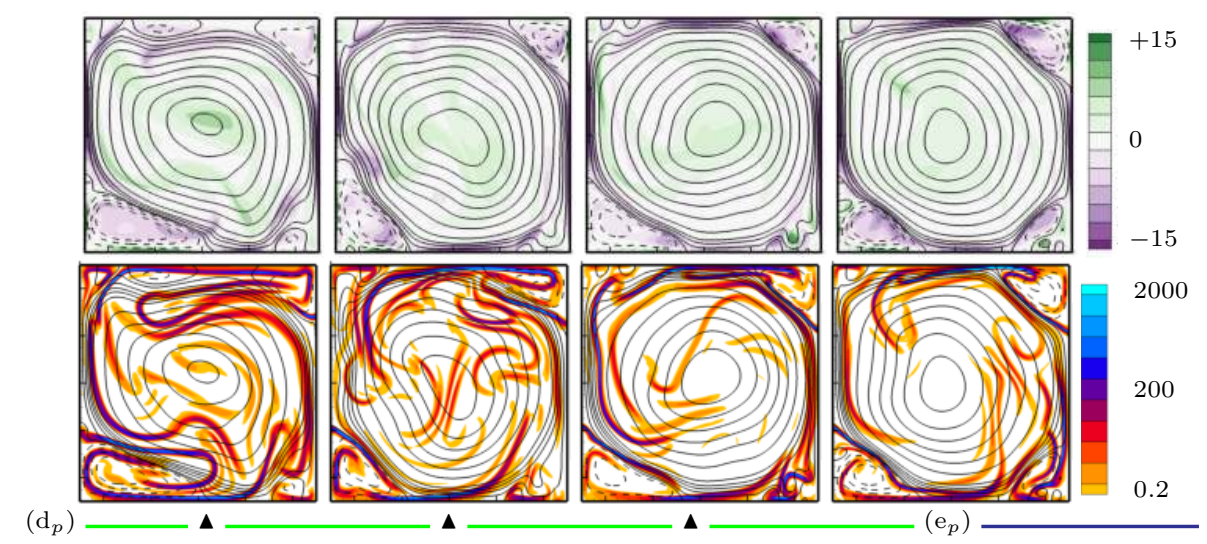

FIGURE 15. Instantaneous fields for a particular reversal cycle taken at regular intervals during the acceleration phase at $\left(\mathrm{Ra}=5 \cdot 10^{7}, \mathrm{Pr}=3.0\right)$. The first snapshot follows point $\left(\mathrm{d}_{p}\right)$; the last corresponds to point $\left(\mathrm{e}_{p}\right)$. Streamlines are superposed either to vorticity $\omega$ (top figures), or to $\nabla y_{r} \cdot \nabla \theta$ (bottom figures).
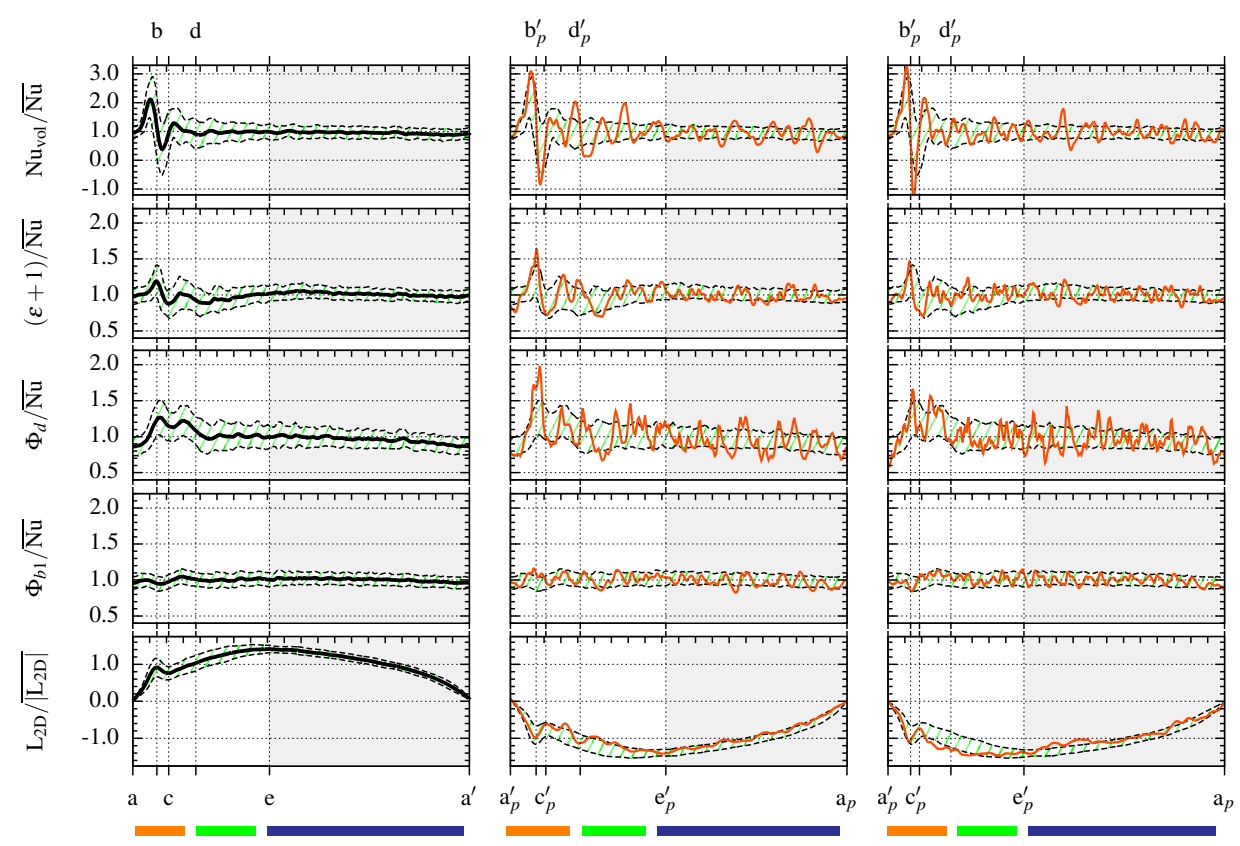

Figure 16. Time evolution of the energy transfer rates. From top to bottom: normalised $\mathrm{Nu}_{\mathrm{vol}} / \overline{\mathrm{Nu}},(\epsilon+1) / \overline{\mathrm{Nu}}, \Phi_{d} / \overline{\mathrm{Nu}},, \Phi_{b 1} / \overline{\mathrm{Nu}}$ for $\left(\mathrm{Ra}=5 \cdot 10^{7}, \operatorname{Pr}=3.0\right)$. Figure for $\mathrm{L}_{2 \mathrm{D}} / \overline{\left|\mathrm{L}_{2 \mathrm{D}}\right|}$ is given as a reference curve. The left figure corresponds the generic reversal: the curve in - corresponds to the average, while the hatched region represent one standard deviation from this average. The center and right figures correspond to particular reversals displayed in alongside the standard deviation.

\subsection{Mechanical energy transfer rates}

Figure 16 displays the evolution of energy transfer rates (given in equations 2.6 to 2.12 ) for a generic reversal cycle as well as for two particular reversal cycles. More precisely, the bulk terms $\mathrm{Nu}_{\mathrm{vol}}=\Phi_{y}+1, \epsilon+1, \Phi_{d}$, and boundary term $\Phi_{b 1}=\frac{1}{2}\left(\mathrm{Nu}_{\mathrm{top}}+\mathrm{Nu}_{\mathrm{bot}}\right)$ normalised by $\overline{\mathrm{Nu}}$ are presented. First, note that, while the time-averaged quantities $\bar{\epsilon}+1$, 


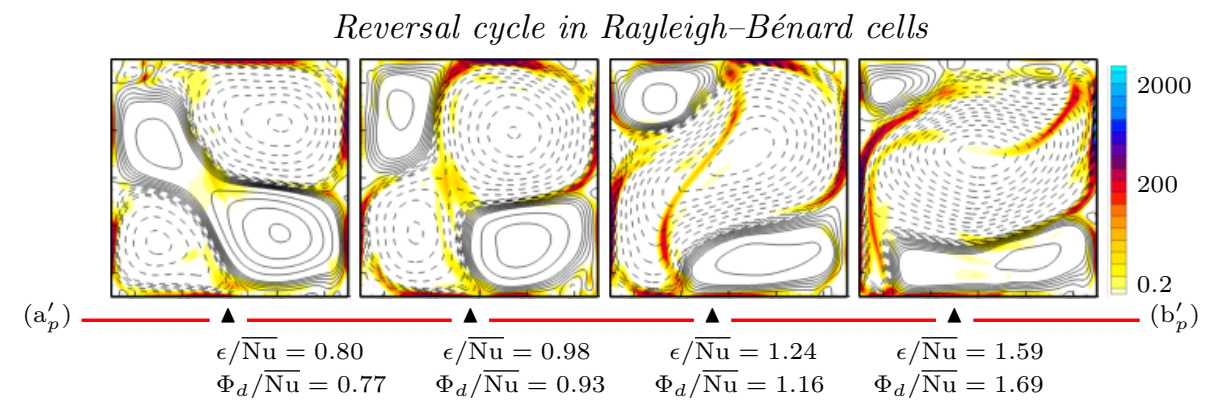

FiguRE 17. Instantaneous fields for a particular reversal cycle taken at regular intervals during the early release phase at $\left(\mathrm{Ra}=5 \cdot 10^{7}, \mathrm{Pr}=3.0\right)$. The first (resp. last) snapshot follows point $\left(a_{p}^{\prime}\right)$ (resp. precedes point $\left.\left(b_{p}^{\prime}\right)\right)$. Streamlines are superposed to field $2 e_{i j} e_{i j}$ the value of which is given by the colour. Corresponding values of $\epsilon$ and $\Phi_{d}$ below each snapshot.

$\overline{\Phi_{d}}$ and $\overline{\Phi_{b 1}}$ converge to $\overline{\mathrm{Nu}}$, each corresponding term has a specific behaviour during the different phases of the reversal cycle. Let us describe each instantaneous transfer rates in turn.

The vertical heat flux $\mathrm{Nu}_{\mathrm{vol}}$ which measures the conversion from $\mathrm{E}_{\text {apot }}$ to $\mathrm{E}_{\text {kin }}$ (see equations 2.7 and 2.8), is by far the term that is found the most fluctuating, notably during the release. During the first part of the rebound i.e. the interval between points $\left(a_{p}\right)$ and $\left(b_{p}\right)$ (resp. (a) and (b)) for the particular reversal (resp. for the generic curve), $\mathrm{Nu}_{\mathrm{vol}}$ reaches peak values which are several times larger than $\overline{\mathrm{Nu}}$. This is related to the release of thermal energy $\mathrm{E}_{\text {apot }}$ (figure 8) and plumes (figure 14). Between points (b) and (c), the generic $\mathrm{Nu}_{\mathrm{vol}}$ abruptly decreases. In terms of a particular realisation, this is due to the rotation of the bulk acting against buoyancy forces (points $\left(b_{p}\right)$ and $\left(c_{p}\right)$ in figure 14) and may result in a negative heat transfer as seen in figure 16 for the particular reversal cycle. This is consistent with results from (Chandra \& Verma 2013). During the acceleration and accumulation phases, $\mathrm{Nu}_{\mathrm{vol}}$ fluctuates less and slightly decreases.

In addition to $\mathrm{Nu}_{\mathrm{vol}}$, the viscous dissipation rate $\epsilon$ governs the evolution of $\mathrm{E}_{\mathrm{kin}}$ (see equation 2.7). Viscous dissipation increases briefly during the re-organisation periods: once during the release phase around point (b), and again during the rotation of the central vortex between points (c) and (d). A gradual increase in $\epsilon$ is observed during the acceleration phase, between points (d) and (e), followed by a progressive decay during the accumulation phase. For a particular reversal cycle, contributions to the viscous dissipation rate $\epsilon$ are located (see figure 17) primarily along the vertical side-walls and along the horizontal plates where ascending (resp. descending) plumes collide.

In addition to $\mathrm{Nu}_{\mathrm{vol}}, \mathrm{E}_{\text {apot }}$ is governed by the mixing term $\Phi_{d}$ (see equation 2.12). As seen in fields $\nabla y_{r} \cdot \nabla \theta$ on figures 14 and 15, contributions to $\Phi_{d}$ are distributed along thin filaments which trace the contour of thermal plumes. $\Phi_{d}$ is thus affected by small scales and fluctuates during all the process.

However, fluctuations are slightly larger during the second part of the release phase (points $\left(b_{p}\right)$ to $\left.\left(d_{p}\right)\right)$. An increase on such mixing fronts during the rotation of the bulk leads to the corresponding increase of $\Phi_{d}$. Note that during the accumulation phase, the generic curve for $\Phi_{d}$ slightly decreases. Finally, the forcing boundary term $\Phi_{b 1}$ does not fluctuate much on the whole cycle.

To summarize, the dominant mechanisms during the release phase are first the energy conversion $\mathrm{Nu}_{\mathrm{vol}}$ followed by the mixing $\Phi_{d}$ during the rebound. The acceleration phase is characterized by the increase of the viscous dissipation $\epsilon$. A constant decay of both dissipation $\epsilon$ and mixing $\Phi_{d}$ processes is conversely observed during the accumulation phase. 

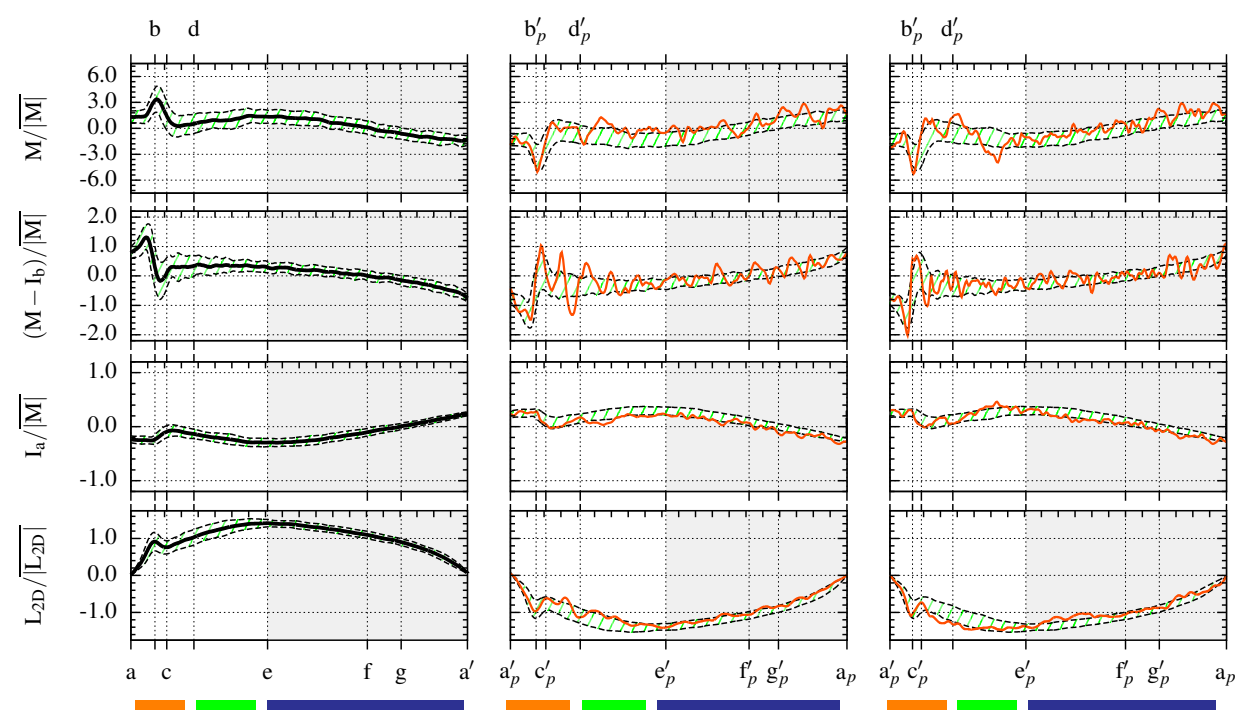

Figure 18. Time evolution of the angular impulse transfer rates for $\left(\mathrm{Ra}=5 \cdot 10^{7}, \mathrm{Pr}=3.0\right)$. From top to bottom: Evolution of $\mathrm{M} / \overline{|\mathrm{M}|},\left(\mathrm{M}-\mathrm{I}_{\mathrm{b}}\right) / \overline{|\mathrm{M}|}$, and $\mathrm{I}_{\mathrm{a}} / \overline{|\mathrm{M}|}$. Figure for $\mathrm{L}_{2 \mathrm{D}} / \overline{\left|\mathrm{L}_{2 \mathrm{D}}\right|}$ is given as a reference curve. The left figure corresponds to the generic reversal: the average curve is displayed in $\longrightarrow$ and the hatched region represents one standard deviation from this average. The center and right figures depict two particular reversal cycles.
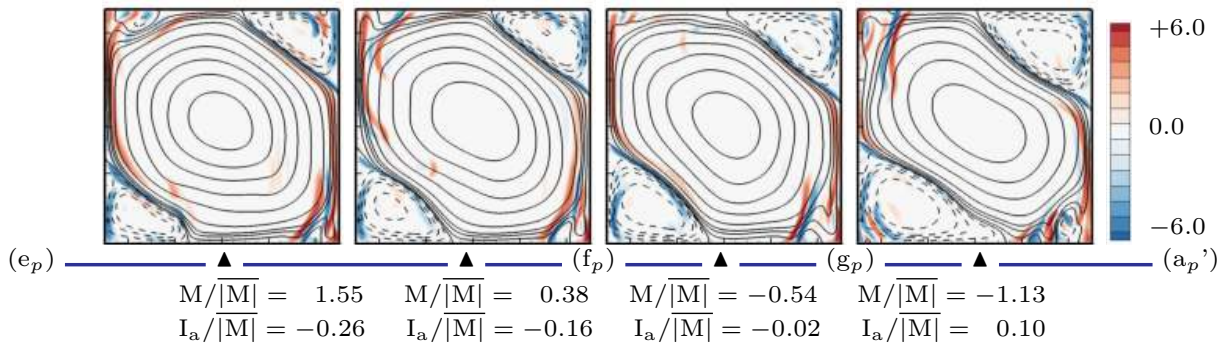

FiguRE 19. Instantaneous fields for a particular reversal cycle taken at regular intervals during the accumulation phase at $\left(\mathrm{Ra}=5 \cdot 10^{7}, \mathrm{Pr}=3.0\right)$. The first (resp. last) snapshot follows point $\left(\mathrm{e}_{p}\right)$ (resp. precedes point $\left(\mathrm{a}_{p}{ }^{\prime}\right)$ ). Streamlines are superposed to field $0.5(\vec{x} \cdot \vec{x}) \partial_{x} \theta$ (the value is provided by the color code). Corresponding values of $\mathrm{M}$ and $\mathrm{I}_{\mathrm{a}}$ are given below each snapshot.

\subsection{Angular momentum transfer rates}

Since the evolution of $\mathrm{L}_{2 \mathrm{D}}$ is characteristic of flow reversals, it is of interest to examine the angular impulse transfer rates $\mathrm{M},\left(\mathrm{M}-\mathrm{I}_{\mathrm{b}}\right)$ and $\mathrm{I}_{\mathrm{a}}$ of equation 2.4 (figure 18). In addition to points (a) to (e), we introduce points (f) and (g). These points are located inside the accumulation phase and coincide for point (f) with a change of sign for $\mathrm{M}$ and for point $(\mathrm{g})$ with a change of sign for $\mathrm{I}_{\mathrm{a}}$.

Time evolution of the input torque $M$ has a maximum value during the rebound, followed by a local minimum near point (d), a slight increase during the acceleration phase and a monotonic decrease during the accumulation phase. The bulk term $\mathrm{M}(t)$ and the boundary term $\mathrm{I}_{\mathrm{b}}(t)$ are well-correlated and have similar orders of magnitude. This is why only the $\mathrm{M}(t)-\mathrm{I}_{\mathrm{b}}(t)$ evolution is plotted.

Before point (e), the difference $\mathrm{M}(t)-\mathrm{I}_{\mathrm{b}}(t)$ is larger than $\mathrm{I}_{\mathrm{a}}(t)$, from (e) to (f) it is the opposite $\left(\frac{\mathrm{dL}_{2 \mathrm{D}}}{\mathrm{dt}}\right.$ changes sign). One may discriminate three time periods: from (a) 
to (f), $\mathrm{I}_{\mathrm{a}}$ opposes $\mathrm{L}_{2 \mathrm{D}}$ (i.e. the central vortex), contrary to the torque $\mathrm{M}$ or $\left(\mathrm{M}-\mathrm{I}_{\mathrm{b}}\right)$; from (f) to (g), $\mathrm{I}_{\mathrm{a}}$ opposes $\mathrm{L}_{2 \mathrm{D}}$ similarly to $\mathrm{M}$ or $\left(\mathrm{M}-\mathrm{I}_{\mathrm{b}}\right)$; and finally, from (g) to (a), $\mathrm{I}_{\mathrm{a}}$ contributes to $\mathrm{L}_{2 \mathrm{D}}$ which is opposed by $\mathrm{M}$ or $\left(\mathrm{M}-\mathrm{I}_{\mathrm{b}}\right)$. During the accumulation phase, the integrand $0.5 \operatorname{Pr}[\vec{x} \cdot \vec{x}] \partial_{x} \theta$ of the input torque $\mathrm{M}(t)$ is spatially distributed as follows (figure 19): it is negligible inside the bulk, and concentrated in two regions, i.e. along vertical side-walls associated with the central vortex and inside corners-flows. In these two regions they are overall of opposite sign. At point (f), $M(t)$ changes sign: the corner-flows become dominant in the integrand of $\mathrm{M}(t)$ and the overall torque is then opposing the central vortex (figure 18). Similarly, during the accumulation phase, the integrand of $I_{a}$ that is vorticity, is distributed in two sectors along the boundaries: one along the boundaries of the central vortex, and another of opposite sign along the top and bottom corners (figure 10). From point (g), $\mathrm{I}_{\mathrm{a}}$ has changed sign: this is related to the dominant contribution of the corner-rolls.

As a consequence, it is noteworthy that the input torque $\mathrm{M}(t)$ changes sign at point (f) long before the reversal time (point (a)). The interval from point (f) to point (g) can be seen as a transition period from a central vortex-dominated to a corner-rolls-dominated flow.

\section{Mechanism of transitions}

\subsection{Linear stability approach}

The significance of instant $(\mathrm{g})$ where $I_{a}$ changes sign, is tentatively explained by two complementary approaches. In a first approach, one considers the following hypothesis: the change occurring in the accumulation phase near point $(\mathrm{g})$, is due to a modification of the dynamics governing fluctuations around large scale structures. The large scale structures identified by fields $\theta^{o}(x, y, t)$ and $\vec{u}^{o}(x, y, t)$ are here obtained by an ensemble average over many realisations (see section 5.1). A linear stability analysis is thus defined. The base state at time $t_{o}$ is given by fields $\theta^{o}\left(x, y, t_{o}\right)$ and $\vec{u}^{o}\left(x, y, t_{o}\right)$ frozen at this particular time. The evolution of infinitesimal fluctuations $\theta^{\prime}(x, y, t)$ and $\vec{u}^{\prime}(x, y, t)$ is studied around this frozen base state. The linear stability analysis is performed by direct numerical simulations of the linearised Boussinesq equations,

$$
\left\{\begin{aligned}
\nabla \cdot \vec{u}^{\prime} & =0 \\
\partial_{t} \vec{u}^{\prime}+\nabla \cdot\left[\vec{u}^{\prime} \otimes \vec{u}^{o}+\vec{u}^{o} \otimes \vec{u}^{\prime}\right] & =-\nabla p^{\prime}+\operatorname{PrRa}^{-0.5} \nabla^{2} \vec{u}^{\prime}+\operatorname{Pr} \theta^{\prime} \vec{e}_{y} \\
\partial_{t} \theta^{\prime}+\nabla \cdot\left[\vec{u}^{\prime} \theta^{o}+\vec{u}^{o} \theta^{\prime}\right] & =\mathrm{Ra}^{-0.5} \nabla^{2} \theta^{\prime}
\end{aligned}\right.
$$

Each linearized simulation starts with random disturbances of velocity and temperature fields and is computed for several hundred time units. The perturbation kinetic energy $\left\langle u_{i}^{\prime} u_{i}^{\prime}\right\rangle_{\text {vol }}$ or the square of the fluctuation temperature $\left\langle\theta^{\prime} \theta^{\prime}\right\rangle_{\text {vol }}$ are monitored in time. In figure 20, each curve is related to a different base state $t_{o}$. Positive values of growth rate $\sigma$ are obtained in all cases.

For $t_{o}$ before transition point $(\mathrm{g})$, the values of $\sigma$ are quite small $\left(\sim 10^{-3}-10^{-2}\right)$ and the most amplified mode corresponds to a trace of the base state itself (see figure bottom left 20). This is possibly related to a slow variation in time of the large scale flow. In contrast, once point $(\mathrm{g})$ is reached, the growth rate $\sigma$ increases by a factor of 20 , while a different most amplified mode appears. This amplified mode is reminiscent of the flow features observed in the bottom right corner of figure 10.

\subsection{Non-linear approach with adiabatic boundary conditions}

We have identified through a linear stability analysis a critical generic state around point (g). Let us now seek to relate this state to the "avalanche" mechanism: a mechanism 
(i) Initial generic condition
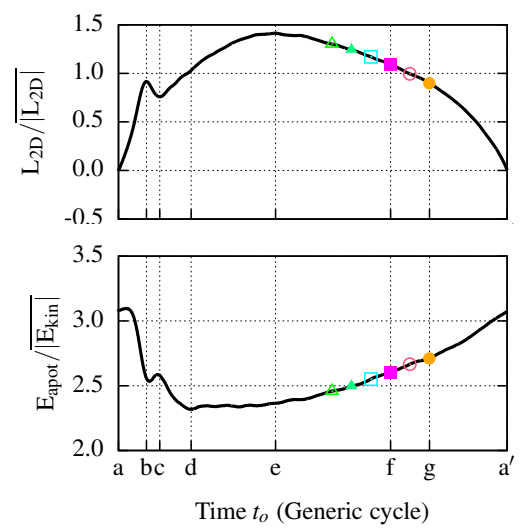

(iii) Base state at time $t_{o}$
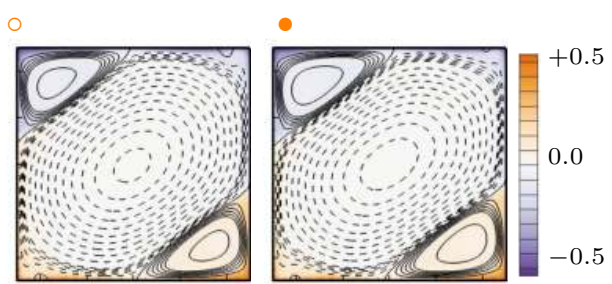

(ii) Normalised perturbation
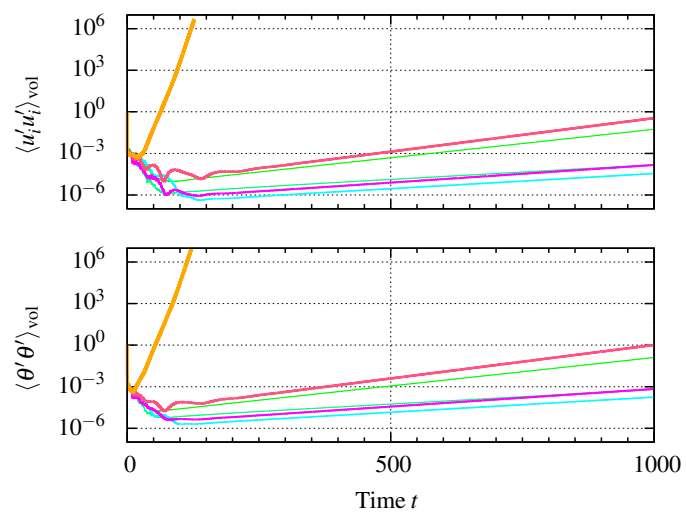

(iv) Disturbance field $\theta^{\prime} \theta^{\prime}(x, y, t)$

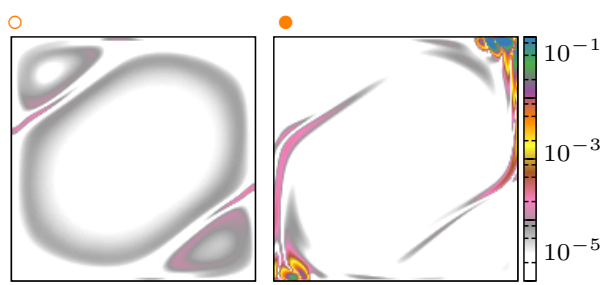

FIGURE 20. Linear stability analysis of the generic fields for $\left(\mathrm{Ra}=5 \cdot 10^{7}, \mathrm{Pr}=3.0\right.$ ). (i) Placement of the different values of $t_{o}$ with respect to the generic $\mathrm{L}_{2 \mathrm{D}}$ and $\mathrm{E}_{\text {apot }}$ curves. (ii) Evolution of the energy of the normalised velocity and temperature perturbations for different base states (or equivalently $\left.t_{o}\right)$. Growth rate $\sigma$ measured for different values of $t_{o}: \Delta \sigma=0.009, \Delta \sigma=0.005$, $\square \sigma=0.005, \square \sigma=0.006, \circ \sigma=0.011, \bullet \sigma=0.250$; (iii) Streamlines corresponding to field $\vec{u}^{o}\left(x, y, t_{o}\right)$ superposed to base state $\theta^{o}\left(x, y, t_{o}\right)$ shown for two values of $t_{o}$ : $\circ$ located between points (f) and (g) and - located on point (g). (iv) Disturbance field $\theta^{\prime} \theta^{\prime}(x, y, t)$ measured at the end of the curves corresponding to $\circ$ and $\bullet$.

due to a localised accumulation of energy inside the fluid which is followed by a sudden transition. One could argue that, when localised accumulation is sufficient, a reversal event takes place even if the external thermal forcing is thereafter suppressed. A second approach, which is non-linear, is based on this idea and confirms point (g) as a transition point associated to a threshold state.

First, let us consider the conditionally averaged fields $\left(\theta^{o}\left(x, y, t_{o}\right)\right.$ and $\left.\vec{u}^{o}\left(x, y, t_{o}\right)\right)$ obtained at time $t_{o}$ for the standard RB problem, see figure 7. Starting from such fields labeled by time $t_{o}$, we perform a direct numerical simulation of the non-linear Boussinesq equations changing boundary conditions from isothermal to adiabatic on the top and bottom plates. This effectively suppresses the external thermal forcing since $\Phi_{b 1}=\Phi_{b 2}=$ 0 and limits the amount of thermal energy contained inside the cavity. Note that, different initial conditions i.e. different $t_{o}$ lead to different trajectories for the adiabatic problem.

Evolution of the normalised kinetic energy $\mathrm{E}_{\mathrm{kin}} / \overline{\left|\mathrm{E}_{\mathrm{kin}}\right|}$ and the normalised angular impulse $\mathrm{L}_{2 \mathrm{D}} / \overline{\left|\mathrm{L}_{2 \mathrm{D}}\right|}$ can be seen in figure 21 , where each curve corresponds to different $t_{o}$ (shown as $\circ$ marks) inside the accumulation phase. Dashed (resp. solid) lines indicate negative (positive) values of $\mathrm{L}_{2 \mathrm{D}} / \overline{\left|\mathrm{L}_{2 \mathrm{D}}\right|}$. For simulations preceding the transition point $(\mathrm{g})$ a decay in both the angular impulse and the kinetic energy are observed. On the contrary, from point $(\mathrm{g})$ on, a change from negative to positive values of $\mathrm{L}_{2 \mathrm{D}} / \overline{\left|\mathrm{L}_{2 \mathrm{D}}\right|}$ simultaneously as peak values in $\mathrm{E}_{\mathrm{kin}} / \overline{\left|\mathrm{E}_{\mathrm{kin}}\right|}$ are observed, before the ensuing decay. To illustrate this 

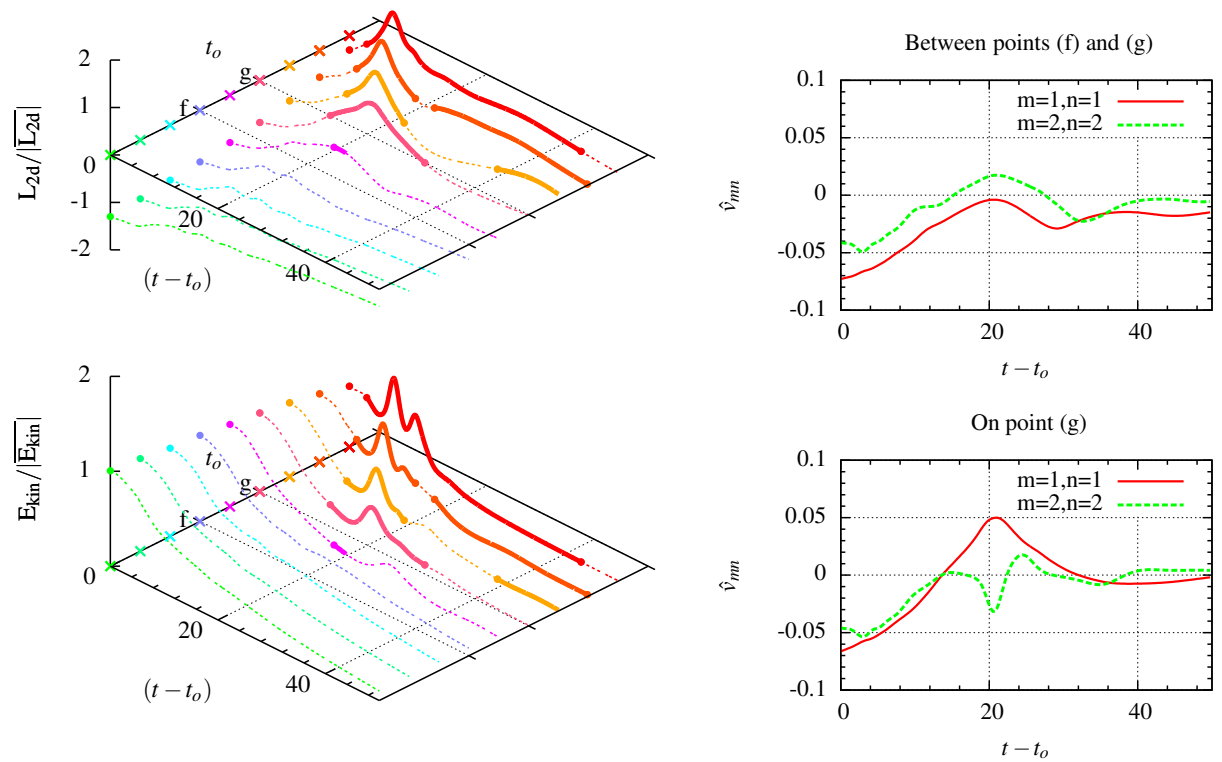

FIGURE 21. Left panel: normalized angular impulse $\left|\mathrm{L}_{2 \mathrm{D}}\right| / \overline{\left|\mathrm{L}_{2 \mathrm{D}}\right|}$ (top) and normalized kinetic energy $\mathrm{E}_{\mathrm{kin}} / \overline{\left|\mathrm{E}_{\mathrm{kin}}\right|}($ bottom $)$ for $\left(\mathrm{Ra}=5 \cdot 10^{7}, \mathrm{Pr}=3.0\right)$ as a function of $t_{o}$ characterizing the base state and time $\left(t-t_{o}\right)$. Each curve represents different initial conditions i.e. different $t_{o}$, where solid lines indicate when a flow reversal takes place (see text). Right panel: Evolution of modal coefficients $\hat{v}_{m n}$ from two initial conditions: before and at point $(g)$.

transition we follow the evolution of Fourier modes $\hat{v}_{m n}$ of the vertical velocity for two initial conditions before and after point $(\mathrm{g})$ as seen on figure 21. For initial conditions preceding $\mathrm{I}_{\mathrm{a}}=0$, an already weakened central vortex is unable to contain the corner-flows in place but does not break (the mode $\hat{v}_{11}$ which is related to the monopole decreases but remains negative). On the contrary, for initial conditions following the transition point (g), Fourier coefficient $\hat{v}_{11}$ changes sign and thus indicates a LSC reversal, before the subsequent decay.

\section{Comparison with previous works}

The present DNS results and their interpretation in terms of dynamical processes may be discussed by comparison with previous experimental data and models. Experimental data (Brown \& Ahlers (2006); Xi \& Xia $(2008 a, b)$ ), and models like (Brown \& Ahlers $(2007,2008))$ are devoted to the study of cylindrical 3D convection cells, where the LSC plane oscillates. They are mostly focused on the dynamics of the angle of the LSC plane which is a different phenomenon from the dynamics studied in this work. Similarly models like (Sreenivasan et al. (2002); Benzi (2005)) which are based on nonlinear one-dimensional stochastic models, have been compared against experimental data in cylindrical cells. Mainly they attempt to recover the exponential distribution for the PDF of the inter-switch intervals. In $2 \mathrm{D}$ systems, such distribution is not observed and again this is probably due to the difference between cessations-driven and rotation-led reversals. Sreenivasan et al. (2002) states that reversals can be understood in terms of an imbalance between buoyancy effects and friction, where inertia is playing only a secondary role. In the present study, reversals do require the accumulation of potential energy and point $(\mathrm{g})$ is associated to a threshold state pinpointed by the change of sign of 
$\mathrm{I}_{\mathrm{a}}$ a quantity related to wall friction. However, the model proposed in Sreenivasan et al. (2002) is a local one, which follows a single parcel of fluid, while our understanding of the dynamics depends on the existence of global flow structures. It is also mentioned in Sreenivasan et al. (2002) the possible role of side-wall thermal conductivity on reversals, which is excluded here. The model by Araujo et al. (2005) is based on the inertia of a plume to initiate the reversal. In our case, inertial effects appear during the rebound: as mentioned in $\S 6.1$, the newly formed circulation is able to advect thermal blobs against the action of buoyancy forces. However, this is a direct consequence of the reversal and not the triggering factor. Low order models could be based also on POD modes (Podvin \& Sergent $(2015,2016))$. This approach allows to rebuild the full dynamics of the velocity and temperature fields. The model proposed in Podvin \& Sergent (2015) that uses 3 leading modes, is able to reproduce large-scale features of our DNS results when noise is introduced: reversal and cessation dynamics, and growth of corner flows during the accumulation phase. It is also able to reproduce the characteristic time scales as given in $\S 4$. However, the phases predominantly associated in the present work to small scales, such as the acceleration and second part of the release, are by construction not recovered by the model described in Podvin \& Sergent (2015). Going back to the threshold state on point (g), the existence of such a point has also been identified through a large scale description of reversals by a POD approach (Podvin \& Sergent (2016)). The scenario of the growth of corner flows leading to the release in the case of a square RB cell has been previously proposed by (Chandra \& Verma (2013); Sugiyama et al. (2010)). Both papers pointed out the feeding of the corner flows by plumes detached from horizontal boundary layers. Our results agree with these findings. But we present this process in a more quantitative way through the use of field $\operatorname{Pr}\left(y_{r}-y\right) \theta$ (see figure 11): in this way, we are able to show that $\mathrm{E}_{\text {apot }}$ is stored mainly in the corner flows. Neither of the two previous papers quantified the energy exchange between kinetic and potential energy during the release. In the present paper, the energy exchange is clearly demonstrated on figures 8 and 9 . Furthermore, the figure 12 shows that the energy exchange takes place between the corner flows and the bulk.

\section{Concluding remarks}

In this paper we used long-term data from two-dimensional DNS in square RB cells inside the $\mathrm{CR}$ regime to perform a statistical characterisation of reversals. Once having removed the samples not contained in the CR regime, a simple time rescaling allowed us to identify a generic reversal cycle in terms of the evolution of three global quantities: the global angular impulse, the global kinetic energy, and available potential energy. Consistent dynamical features were found for different values of (Ra, Pr), and suggested the existence of a generic reversal mechanism, composed of three successive phases: accumulation, release, and acceleration.

The accumulation phase is characterised by a progressive build-up of thermal energy almost exclusively inside the corners thereby inducing them to grow. During the release phase, an energy exchange takes place from available potential energy to global kinetic energy: the opposing corner-flows connect to form a new central vortex and the thermal energy contained from small-scales is suddenly released into the bulk. A newly formed vortex may complete several turnovers before the temperature differences inside the bulk are reduced. Strong fluctuations in both the angular impulse and the kinetic energy are observed during this process, referred to as a rebound. During the acceleration phase, the global angular impulse and the kinetic energy increase as a result of spontaneous self-organisation of the flow into a large diagonal vortex and two small counter-rotating 
corner-flows. During this phase, increased mixing inside the central vortex results in a very homogeneous bulk temperature and almost constant potential energy. We complement this view in terms of the evolution of energy transfer rates.

Finally, in order to identify the presence of a transition between accumulation and release, we propose two approaches. First a linear stability analysis is performed around generic fields $\vec{u}^{o}\left(x, y, t_{o}\right)$ and $\theta^{o}\left(x, y, t_{o}\right)$ (obtained as the ensemble average over long term DNS results). A sharp increase in the exponential growth rate $\sigma$ is shown before the beginning of the release phase. In a second approach, the same transition zone is put into evidence by suppressing the external thermal forcing and letting the system evolve from different initial conditions inside the accumulation phase. The presence of a reversal-type event is an indication that a sufficient amount of thermal energy already being stored in the system triggers the reversal.

This work can be easily extended for $2 \mathrm{D}$ cells with different geometries or different boundary conditions. This could provide a complementary view to improve the understanding of reversals in $2 \mathrm{D}$ convection, in particular to the role of corner flows. In a future work, we intend to compare more precisely the present findings with results from PODbased models proposed in Podvin \& Sergent (2015, 2016). In RB 3D cells, the energy budget has already been used but it was considered only in a time-averaged sense. The instantaneous energy budget used here and the way it is related to flow structures can be extended to 3D cells. Indeed, this approach is currently being implemented by the authors in the particular case of a $3 \mathrm{D}$ cell which is very much confined in the transversal direction. Our statistical approach however might be difficult to use in cylindrical convection cells for two reasons: first, one needs a long time signal containing a sufficient number of events. This is a difficult thing to achieve in a fully resolved 3D DNS. Second, the situation is more complex in $3 \mathrm{D}$ than $2 \mathrm{D}$ since in cessation-led (resp. rotation-led) reversals, the LSC plane changes in time abruptly (resp. through azimuthal or torsional motions). This implies to identify or develop a new criteria to follow the evolution of the LSC. This is a limiting factor for expanding the method to $3 \mathrm{D}$ convection case in a cylindrical domain.

\section{Acknowledgements}

This work was granted access to the HPC resources of GENCI-IDRIS under allocations 2014- and 2015- 2a0326 made by GENCI. We acknowledge fruitful discussions with C. Nore, B. Podvin, and M.K. Verma.

\section{REFERENCES}

Araujo, Francisco Fontenele, Grossmann, Siegfried \& Lohse, Detlef 2005 Wind reversals in turbulent Rayleigh-Bénard convection. Physical Review Letters 95, 084502.

Bailon-Cuba, J., Emran, M. S. \& Schumacher, J. 2010 Aspect ratio dependence of heat transfer and large-scale flow in turbulent convection. Journal of Fluid Mechanics 655, $152-173$.

Bell, JB, Colella, P \& Glaz, Hm 1989 A second-order projection method for the incompressible Navier-Stokes equations. Journal of Computational Physics 283, 257-283.

Benzi, Roberto 2005 Flow reversal in a simple dynamical model of turbulence. Physical Review Letters 95 (2), 024502.

Brown, Eric \& Ahlers, Guenter 2006 Rotations and cessations of the large-scale circulation in turbulent Rayleigh-Bénard convection. Journal of Fluid Mechanics 568, 351-386.

Brown, Eric \& Ahlers, Guenter 2007 Large-scale circulation model for turbulent RayleighBénard convection. Physical Review Letters 98 (13), 134501.

Brown, Eric \& Ahlers, Guenter 2008 A model of diffusion in a potential well for the 
dynamics of the large-scale circulation in turbulent Rayleigh-Bénard convection. Physics of Fluids (1994-present) 20 (7), 075101.

Chandra, Mani \& Verma, Mahendra K 2011 Dynamics and symmetries of flow reversals in turbulent convection. Physical Review E 83 (6), 067303.

Chandra, Mani \& Verma, Mahendra K. 2013 Flow reversals in turbulent convection via vortex reconnections. Physical Review Letters 110, 114503.

Clercx, HJH, Maassen, SR \& Van HeiJst, GJF 1998 Spontaneous spin-up during the decay of $2 \mathrm{~d}$ turbulence in a square container with rigid boundaries. Physical Review Letters $80(23), 5129$.

Funfschilling, Denis, Brown, Eric \& Ahlers, Guenter 2008 Torsional oscillations of the large-scale circulation in turbulent Rayleigh-Bénard convection. Journal of Fluid Mechanics 607, 119-139.

Grossmann, Siegfried \& Lohse, Detlef 2003 On geometry effects in Rayleigh-Bénard convection. Journal of Fluid Mechanics 486, 105-114.

Hughes, Graham O., Gayen, Bishakhdatta \& Griffiths, Ross W. 2013 Available potential energy in Rayleigh-Bénard convection. Journal of Fluid Mechanics 729, R3.

Lhuillier, Florian, Hulot, Gauthier \& Gallet, Yves 2013 Statistical properties of reversals and chrons in numerical dynamos and implications for the geodynamo. Physics of the Earth and Planetary Interiors 220, 19-36.

LORENZ, EDWARD N 1955 Available potential energy and the maintenance of the general circulation. Tellus 7 (2), 157-167.

Molenaar, D., Clercx, H. J H \& Van Heijst, G. J F 2004 Angular momentum of forced 2D turbulence in a square no-slip domain. Physica D: Nonlinear Phenomena 196, 329-340.

Ni, Rui, Huang, Shi-Di \& XiA, Ke-Qing 2015 Reversals of the large-scale circulation in quasi-2D Rayleigh-Bénard convection. Journal of Fluid Mechanics 778, R5.

Niemela, JJ, Skrbek, L, Sreenivasan, KR \& Donnelly, RJ 2001 The wind in confined thermal convection. Journal of Fluid Mechanics 449, 169-178.

Petschel, K, Wilczek, M, Breuer, M, Friedrich, R \& Hansen, U 2011 Statistical analysis of global wind dynamics in vigorous Rayleigh-Bénard convection. Physical Review E 84 (2), 026309.

Podvin, Bérengère \& Sergent, Anne 2015 A large-scale investigation of wind reversal in a square Rayleigh-Bénard cell. Journal of Fluid Mechanics 766, 172-201.

Podvin, Bérengère \& Sergent, Anne 2016 On a precursor mechanism for wind reversal in a square Rayleigh-Bénard cell. submitted .

van der Poel, Erwin P., Stevens, Richard J. A. M. \& Lohse, Detlef 2011 Connecting flow structures and heat flux in turbulent Rayleigh-Bénard convection. Physical Review E 84, 045303.

Popinet, S. 2016 Basilisk. Website, http://basilisk.fr.

Resagk, Christian, du Puits, Ronald, Thess, André, Dolzhansky, Felix V., Grossmann, Siegfried, Fontenele Araujo, Francisco \& Lohse, Detlef 2006 Oscillations of the large scale wind in turbulent thermal convection. Physics of Fluids 18 (9), 095105.

Shraiman, Boris I. \& Siggia, Eric D. 1990 Heat transport in high-Rayleigh-number convection. Physical Review A 42, 3650-3653.

Sreenivasan, Katepalli R, Bershadskit, Alexander \& Niemela, JJ 2002 Mean wind and its reversal in thermal convection. Physical Review E 65 (5), 056306.

Stevens, Richard J. A. M., Verzicco, Roberto \& Lohse, Detlef 2010 Radial boundary layer structure and Nusselt number in Rayleigh-Bénard convection. Journal of Fluid Mechanics 643, 495-507.

Sugiyama, Kazuyasu, Ni, Rui, Stevens, Richard J. A. M., Chan, Tak Shing, Zhou, Sheng-Qi, Xi, Heng-Dong, Sun, Chao, Grossmann, Siegrried, Xia, Ke-Qing \& Lohse, Detlef 2010 Flow reversals in thermally driven turbulence. Physical Review Letters 105, 034503.

Sutherland, Bruce R 2010 Internal gravity waves. Cambridge University Press.

Tseng, Yu Heng \& Ferziger, Joel H. 2001 Mixing and available potential energy in stratified flows. Physics of Fluids 13 (5), 1281-1293.

Valet, Jean-Pierre, Fournier, Alexandre, Courtillot, Vincent \& Herrero-Bervera, 
EMiLIo 2012 Dynamical similarity of geomagnetic field reversals. Nature 490 (7418), 8993.

Verma, Mahendra K., Ambhire, Siddhesh C. \& Pandey, Ambrish 2015 Flow reversals in turbulent convection with free-slip walls. Physics of Fluids 27 (4), 047102.

Wagner, Sebastian \& Shishkina, Olga 2013 Aspect-ratio dependency of Rayleigh-Bénard convection in box-shaped containers. Physics of Fluids 25 (8), 085110.

Wicht, Johannes, Stellmach, Stephan \& Harder, Helmut 2009 Numerical models of the geodynamo: From fundamental cartesian models to 3D simulations of field reversals. In Geomagnetic Field Variations, pp. 107-158. Springer Berlin Heidelberg.

Winters, Kraig B., Lombard, Peter N., Riley, James J. \& D'Asaro, Eric A. 1995 Available potential energy and mixing in density-stratified fluids. Journal of Fluid Mechanics 289, 115-128.

XI, Heng-Dong \& Xia, Ke-Qing 2008 a Azimuthal motion, reorientation, cessation, and reversal of the large-scale circulation in turbulent thermal convection: a comparative study in aspect ratio one and one-half geometries. Physical Review E 78 (3), 036326.

XI, Heng-Dong \& XIA, Ke-QING $2008 b$ Flow mode transitions in turbulent thermal convection. Physics of Fluids 20 (5), 055104.

Xia, Ke-Qing, Sun, Chao \& Zhou, Sheng-Qi 2003 Particle image velocimetry measurement of the velocity field in turbulent thermal convection. Physical Review E 68, 066303. 